\title{
1 PLZF is a new substrate of CRBN with thalidomide and 5-
}

\section{2 hydroxythalidomide}

3 Satoshi Yamanaka ${ }^{1}$, Hidetaka Murai ${ }^{2}$, Daisuke Saito ${ }^{2 \#}$, Gembu Abe $^{2}$, Etsuko Tokunaga ${ }^{3}$,

4 Takahiro Iwasaki ${ }^{4}$, Hirotaka Takahashi $^{1}$, Hiroyuki Takeda $^{4}$, Takayuki Suzuki $^{5}$, Norio

$5 \quad$ Shibata $^{3}$, Koji Tamura $^{2} \&$ Tatsuya Sawasaki ${ }^{*}$

$6{ }^{1}$ Division of Cell-Free Sciences, Proteo-Science Center, Ehime University, Matsuyama,

7 790-8577 Japan, ${ }^{2}$ Department of Ecological Developmental Adaptability Life Sciences,

8 Graduate School of Life Sciences, Tohoku University, Sendai, 980-8578, Japan,

$9 \quad{ }^{3}$ Department of Nanopharmaceutical Sciences, Nagoya Institute of Technology, Nagoya,

10 466-8555, Japan, " Division of Proteo-Drug-Discovery Sciences, Proteo-Science Center,

11 Ehime University, Matsuyama, 790-8577 Japan. ${ }^{5}$ Avian Bioscience Research Center,

12 Graduate School of Bioagricultural Sciences, Nagoya University, Nagoya, 464-8601,

13 Japan.

14 \#Present address.

15 Department of Biology, Faculty of Sciences, Kyushu University, Fukuoka, 819-0395 Japan.

16

17 *Corresponding Author:

18 Tatsuya Sawasaki

19 Proteo-Science Center,

20 Ehime University, Matsuyama 790-8577, Japan

21 Tel: $81-89-927-8530$

22 Fax: 81-89-927-9941

23 E-mail address: sawasaki@ehime-u.ac.jp. 
Abstract

Thalidomide induces cereblon (CRBN)-dependent degradation of proteins. Human cytochrome P450s are thought to provide two monohydroxylated metabolites from thalidomide, and the metabolites are also considered to be involved in thalidomide effects. However, it remains unclear. We report that human PLZF/ZBTB16 is a target protein of CRBN with thalidomide and its derivatives, and that 5-hydroxythalidomide has high potential for degrading PLZF. Using a human transcription factor protein array produced by a wheat cell-free protein synthesis system, PLZF was found to bind to CRBN with thalidomide. PLZF is degraded by the CRL4 ${ }^{\text {CRBN }}$ complex with thalidomide and its derivatives. Mutagenesis analysis revealed that both 1st and 3rd zinc finger domains conserved in vertebrates are recognized for thalidomide-dependent binding and degradation by CRBN. In chicken limbs, knockdown of Plzf induced skeletal abnormalities, and Plzf was degraded after thalidomide or 5-

38 hydroxythalidomide treatment. Our findings suggest that PLZF is a pivotal substrate involving thalidomide-induced teratogenesis. 


\section{Introduction}

42 In many countries, thalidomide (Fig. 1a) was widely used to treat morning sickness in

43 pregnant women, and caused embryopathies such as limb defects, ear damage, and congenital

44 heart diseases ${ }^{1-3}$. Handa's group in Japan found that thalidomide binds to the cereblon

45 (CRBN) protein within the CRL4 E3 ubiquitin ligase complex and that CRBN is a key

46 molecule for thalidomide-induced teratogenesis ${ }^{4}$. Recently many studies have reported that

47 CRBN, by interacting with thalidomide or its derivatives, lenalidomide, pomalidomide (Fig.

48 1a), and CC-885, changes its binding specificity to proteins, then induces ubiquitination and

49 degradation of binding proteins such as Ikaros (IKZF1) $)^{5,6}$, casein kinase $\mathrm{I}^{7}$, GSPT1 ${ }^{8}$, and

50 SALL4 ${ }^{9,10}$. In humans, mutations of SALL4 are found in Duane-radial ray syndrome (DRRS,

51 Okihiro syndrome), which has phenotypic features such as limb deformities ${ }^{11}$. In Sall4-

52 conditional knockout (SALL4-CKO) mice, hindlimb defects are also reported ${ }^{12}$. These

53 findings strongly suggest that SALL4 is partially involved in teratogenesis. However, it was

54 reported that forelimb of SALL4-CKO mice were not abnormality and posterior of hindlimb

55 were formed in the SALL4-CKO mice. Furthermore, although thalidomide embryopathy

56 occurs in chicken and zebrafish ${ }^{4}$, the sequence of thalidomide-binding sites in these Sall4s is

57 quite different between human and these animals ${ }^{9}$, suggesting the possibility of other target

58 proteins for teratogenesis.

59 Promyelocytic leukaemia zinc finger (PLZF), also known as ZBTB16 or ZFP145, is a

60 transcription factor (TF) that has nine $\mathrm{C}_{2} \mathrm{H}_{2}$-type zinc finger domains $(\mathrm{ZNFs})^{13}$, and is

61 involved in a broad range of developmental and biological processes, such as haematopoiesis,

62 limb skeletal formation, spermatogenesis, and immune regulation ${ }^{13-15}$. Loss of PLZF function

63 in both human patients and mouse mutants indicates limb defects, which mice phenotype was 
64 elongation defect of zeugopod and thumb ${ }^{14-16}$. A recent study has indicated that the 6th and

657 th ZNFs in PLZF were not targets of $\mathrm{CRBN}^{17}$. However, it remains unknown whether full-

66 length PLZF protein is a target for CRBN with thalidomide.

Thalidomide is metabolized into 5-hydroxythalidomide and 5'-hydroxythalidomide by

68

69 several types of human cytochrome P450 (CYP) ${ }^{18-20}$, which are oxidized in phthalimido and glutarimide rings, respectively (Fig. 4a). Because CRBN mainly recognizes the glutarimide ring in thalidomide $21,22,5^{\prime}$-hydroxythalidomide has no functions in either CRBN binding or teratogenesis ${ }^{23,24}$. Recently, mice having humanized-CYP3A that could potentially provide 5-hydroxythalidomide were shown to undergo teratogenesis after thalidomide treatment ${ }^{25}$, suggesting that 5-hydroxythalidomide functions like thalidomide. However, there is no evidence to suggest whether 5-hydroxythalidomide induces interactions between CRBN and substrate proteins.

We aimed to identify the protein that binds thalidomide-dependently to CRBN. For this, we constructed a human TF protein array (HuTFPA) consisting of 1,118 human recombinant proteins including mainly TFs and zinc finger proteins (Supplementary Table 1), which was produced using a wheat cell-free protein production system. Biochemical screening based on an interaction between $\mathrm{TF}$ and $\mathrm{CRBN}$ with thalidomide using the AlphaScreen system, identified PLZF as an interactor of CRBN with thalidomide. PLZF was shown to be a novel substrate of the CRL4 ${ }^{\mathrm{CRBN}}$ E3 ubiquitin ligase complex with thalidomide, its derivatives, and 5-hydroxythalidomide. Amino acid sequences of PLZF were very similar among vertebrates. In the chick embryo, knockdown of Plzf/Zbtb16 induced abnormal limb development. More importantly, Plzf protein was decreased in the limb buds during thalidomide-induced teratogenesis, whereas expression level of Sall4 protein was not changed. 


\section{Results}

89

90

\section{Screening for thalidomide-dependent substrates of CRBN using a human transcription} factor protein array.

Many TFs function as master regulators during development and differentiation of embryos. In addition, many substrates of CRBN with thalidomide were ZNF-type TFs such as IKZF $1^{5,6}, \mathrm{IKZF}^{5,6}$, and SALL4 ${ }^{9,10}$. We aimed to identify new substrates of CRBN with thalidomide from human TFs. Based on a wheat cell-free protein synthesis system ${ }^{26}$, we had previously developed a technology for the construction of a protein array that synthesizes an individual protein in each well of a 96- or 384-well plate, and have published many reports regarding the identification of substrate proteins of protein kinase ${ }^{27}$ and E3 ligase $^{28,29}$ protein arrays. The combination of our protein array and AlphaScreen technology provides several advantageous features for the screening of protein-protein interactions: it is 1) used directly without protein purification, 2) highly sensitive, and 3) a high-throughput system. The human TF protein array (HuTFPA) consisting of mainly human TFs (Supplementary Table 1), synthesized as N-terminal FLAG-GST fusions, produced by a wheat cell-free system. CRBN was synthesized as an N-terminal single biotin-labelled form, using the same system. The principle of detecting this biochemical interaction is shown in Fig. 1b. Using this cell-free system, an interaction between FLAG-GST-IKZF1 and biotinylated CRBN was thalidomide dose-dependently detected using the AlphaScreen method (Fig. 1c). As shown in the flowchart (Supplementary Fig. 1a), we screened the substrate human TFs of CRBN with thalidomide $(50 \mu \mathrm{M})$ on the HuTFPA, identifying six TF proteins as being CRBN binding proteins in the presence of thalidomide (Fig. 1d). In contrast, several known substrates such 
110 as IKZF3 and CK1 $\alpha$, were not detected because these substrates were not included in the 111 HuTFPA (Supplementary Table 1).

112 To investigate the thalidomide dependency of these proteins for CRBN binding, the 113 biochemical assay was carried out with and without thalidomide. Three human TFs, IKZF1, 114 SALL4, and PLZF, indicated characteristics of thalidomide-dependent binding to CRBN (Fig. 115 1e), whereas HNRNPK, HMGB2, and ELF5 bound to CRBN without thalidomide.

116 Furthermore, in the presence of thalidomide, these did not bind to mutant CRBN-YW/AA ${ }^{3}$ 117 that is unable to bind to thalidomide (Supplementary Fig. 1b). Using recombinant proteins, 118 in vitro pull-down assay confirmed that PLZF binds to wild-type (WT) CRBN in the presence 119 of thalidomide (Supplementary Fig. 1c), as do IKZF1 and SALL4, whereas this binding was 120 not observed under the condition of using mutant CRBN-YW/AA (MT) and no addition of 121 thalidomide (-). These results indicate that PLZF is an interactor of CRBN with thalidomide.

\section{PLZF binds to CRBN with thalidomide, pomalidomide, and lenalidomide.}

124 with thalidomide. PLZF is classified as being part of the ZBTB (zinc finger and bric à brac, tramtrack, and broad) protein family ${ }^{13}$. Since the other ZBTB proteins are included in the

126 HuTFPA, these were analysed for interactions with or without thalidomide. However, the

127 ZBTB proteins did not bind to CRBN with thalidomide (Supplementary Fig. 1d), suggesting 128 that CRBN with thalidomide recognizes a specific region(s) in PLZF, but not one common 129 to the ZBTB family.

130 During the last two decades, two thalidomide derivatives, lenalidomide and pomalidomide 131 (Fig. 1a), have been developed for multiple myeloma (MM) or as immunomodulatory drugs 
132 (IMiDs) ${ }^{30}$. Recent studies have reported that some proteins have different preferences

133 between thalidomide and its derivatives for binding to $\mathrm{CRBN}^{7,9}$. For an example, a CK1 $\alpha-$

134 CRBN interaction is enabled by lenalidomide, but not thalidomide or pomalidomide ${ }^{7}$. We

135 therefore investigated the biochemical characteristics of interactions between PLZF and

136 CRBN with thalidomide and its two derivatives. As a result, thalidomide, pomalidomide and

137 lenalidomide induced PLZF-CRBN interaction, and it is showed that the biochemical

138 binding potency is pomalidomide $>$ lenalidomide $>$ thalidomide (Fig. 1f). In a previous report ${ }^{9}$,

139 it was showed that thalidomide and its two derivatives induced degradation of SALL4. In

140 addition, in vitro binding assay using the AlphaScreen method confirmed that SALL4's

141 binding potency is pomalidomide>thalidomide>lenalidomide (Supplementary Fig. 1e).

142 Because the binding potency of PLZF-thalidomide is similar to that of SALL4-lenalidomide,

143 it was predicted that PLZF and SALL4 are pan-substrates on thalidomide and its two 144 derivatives.

\section{PLZF is degraded by the CRL4 ${ }^{\text {CRBN }}$ complex with thalidomide and its derivatives.}

148 RBX1, and CUL4 ${ }^{4}$. Therefore, the PLZF-CRBN interaction with thalidomide and its two

149 derivatives is expected to lead to degradation of PLZF. For a cell-based immunoblotting assay, 150 we used the AGIA-tag system because it is a highly-sensitive tag based on a rabbit 151 monoclonal antibody ${ }^{31}$. To investigate the stability of PLZF or SALL4, AGIA-tagged PLZF 152 or SALL4 was transfected into HEK293T cells with thalidomide, pomalidomide, and 153 lenalidomide. These compounds all decreased the stability of PLZF and SALL4 154 (Supplementary Fig. 2a). In addition, to demonstrate whether PLZF is also pan-substrate, 
155

156

157

158

159

160

161

162

163

164

165

166

167

168

169

170

171

172

173 174 not degrade AGIA-PLZF in the presence of lenalidomide (Supplementary Fig. 2e),

175 suggesting the CRBN-dependent degradation of PLZF.

176

76 We next investigated whether PLZF is recruited to the CRL4 ${ }^{\text {CRBN }}$ complex and
77 ubiquitinated. By immunoprecipitation of FLAG-CRBN using an anti-FLAG antibody, the

176 We next investigated whether PLZF is recruited to the CRL4 ${ }^{\text {CRBN }}$ complex and
177 ubiquitinated. By immunoprecipitation of FLAG-CRBN using an anti-FLAG antibody, the

such as SALL4, endogenous PLZF or SALL4 protein levels were examined in HuH7 or HEK293T cells treated with thalidomide and its two derivatives. Immunoblot analyses revealed that endogenous PLZF were destabilized by all of them in both cell lines (Supplementary Fig. 2b and c), indicating that PLZF is also pan-substrate on thalidomide and its two derivatives. To reduce experimental complexity, therefore, we focused thalidomide and lenalidomide in further analyses. A remarkable decrease in PLZF was observed 6 hours after lenalidomide treatment (Supplementary Fig. 2d). This time course analysis suggests that the reduction in PLZF was late as compared to other CRBN substrates because degradations of IKZF1, IKZF3, and SALL4 were observed only 3 hours after the treatment ${ }^{5,6,9}$. The lenalidomide-dependent destabilisation of PLZF was completely inhibited by the proteasome inhibitor MG132 and the NEDD8 inhibitor MLN4924 (Fig. 2a), suggesting that PLZF is ubiquitinated by the CRL4 complex for degradation by the proteasome.

To investigate whether the destabilisation of PLZF is dependent on CRBN, we made CRBN-deficient HEK293T cells using CRISPR/Cas9. In the presence of lenalidomide, degradation of endogenous PLZF was observed in normal HEK293T cells, whereas endogenous PLZF was not degraded in CRBN-deficient cells (Fig. 2b). Endogenous PLZF protein was also reduced by lenalidomide in HuH7 and THP-1 cells (Supplementary Fig. 3b and c). Expression of endogenous PLZF mRNA in these cells was unaffected by lenalidomide treatment (Supplementary Fig. 3a-c). In addition, the FLAG-CRBN mutant (YW/AA) did 
178 CRL4 ${ }^{\mathrm{CRBN}}$ complex consisting of DDB1, RBX1, and CUL4 was pulled-down. At the same 179 time AGIA-tagged PLZF was also strongly associated with the complex after thalidomide or 180 lenalidomide treatment (Fig. 2c and Supplementary Fig. 4a), indicating that PLZF is 181 thalidomide- or its two derivatives-dependently included in the CRL4 ${ }^{\text {CRBN }}$ complex. In addition, to analyse the ubiquitination of PLZF, AGIA-PLZF was transfected with FLAG-

183 CRBN and immunoprecipitated for immunoblotting. The smear band resulting from the 184 immunoprecipitation of AGIA-PLZF was increased by supplementation with thalidomide or 185 lenalidomide, suggesting that PLZF ubiquitination was induced by thalidomide and its two 186 derivatives treatment (Fig. $2 d$ and Supplementary Fig. 4b). Next, to investigate the in vitro 187 ubiquitination of PLZF, CRL4 $4^{\text {FLAG-CRBN }}$ and AGIA-PLZF were coimmunoprecipitated by 188 anti-FLAG antibody in the presence or absence of lenalidomide (lane 5-8 in Fig. 2e). As 189 negative control, empty vector or AGIA-PLZF expressing HEK293T cells were lysed and 190 mixed, and the lysates were immunoprecipitated by anti-AGIA antibody to demonstrate the 191 ubiquitination of PLZF caused by CRL4 ${ }^{\text {FLAG-CRBN }}$ (lane 1-4 in Fig. 2e). When 192 coimmunoprecipitating PLZF and CRBN plus exogenous E1 and E2 enzymes, PLZF was 193 ubiquitinated in the presence of lenalidomide (lane 8 in Fig. 2e), but not in its absence (lane 1946 in Fig.2e), indicating that CRL4 ${ }^{\text {FLAG-CRBN }}$ ubiquitinates PLZF in vitro. Taken together, these 195 results show that PLZF is a target of the CRL4 ${ }^{\mathrm{CRBN}}$ complex for proteasome degradation.

Next, because it was reported that PLZF play important role in immune responses ${ }^{13}$, 197 we investigated whether degradation of PLZF is also caused in lymphoma cell lines. 198 Immunoblot analyses showed that IMiD treatment induced protein degradation of PLZF in 199 ABC-DLBCL (TK), GCB-DLBCL (BJAB and HT), Adult T-cell lymphoma/ATL (MT-4), 200 and Burkitt's lymphoma (Raji) cell lines (Supplementary Fig. 5a and b). In GCB-DLBCL 201 (SU-DHL-4) cells, IMiD treatment scarcely induced protein degradation of PLZF but protein 
expression of CRBN in SU-DHL-4 cells was very weak (Supplementary Fig. 5a). These results strongly suggest that PLZF degradation is caused by IMiD treatment in various cells.

Both ZNF1 and ZNF3 domains in PLZF are recognized for its thalidomide-dependent interaction with CRBN.

We next attempted to determine the thalidomide-dependent CRBN interaction region within PLZF. PLZF has a single BTB domain and nine $\mathrm{ZNFs}^{13}$ (Fig. 3a). A single ZNF domain present in $\mathrm{IKZF}^{17}$ and SALL4 ${ }^{9,10}$ is recognized by CRBN with thalidomide. Expectedly, the BTB domain alone in PLZF did not induce CRBN binding (Fig. 3b). We thus

211 constructed a total of five clones lacking different ZNFs by N-terminal FLAG-GST-fusions,

212 then measured the interaction signals between each clone and CRBN with thalidomide. As a

213 result, a ZNF1-5 clone indicated sufficient binding ability to CRBN with thalidomide (Fig.

$2143 \mathrm{~b}$ ). To refine the key domains on the native form of PLZF, each of the five ZNFs were 215 individually swapped with ZNF7 (Fig. 3c), as this had no effect on binding ${ }^{17}$. These binding 216 assays showed that the ZNF1 and ZNF3 domains were most important for binding (Fig. 3d).

217 In previous reports, a glycine residue in a ZNF domain of SALL4 and ZFP91 proteins was 218 shown to be a key amino acid for binding to $\mathrm{CRBN}$ with thalidomide ${ }^{9,10,32}$. We thus made 219 mutant clones (Gly to Ala shown in Fig. 3e) having a single or double substitution in ZNF1 220 and ZNF3, which were then analysed in biochemical (Fig. 3f) and cell-based (Fig. 3g) assays.

221 Surprisingly, the double mutation completely lost the ability for binding and degradation, 222 whereas binding ability of both single mutants significantly low but retained degradation 223 ability (Fig. 3f and g). Taken together, these results indicate that both ZNF1 and ZNF3 224 domains in PLZF are recognized for binding and degradation by CRBN with thalidomide. 


\section{5-hydroxythalidomide induces degradation of PLZF and SALL4, but not of IKZF1.}

229 unclear. We thus investigated whether it induces a CRBN-protein interaction. Surprisingly, 230 5-hydroxythalidomide induces CRBN-PLZF and CRBN-SALL4 interactions (Fig. 4b), 231 whereas it did not induce an interaction between CRBN and IKZF1. Furthermore, 5232 hydroxythalidomide induces the degradation of PLZF and SALL4 in cells, but not of IKZF1 233 (Fig. 4c). Endogenous PLZF and SALL4 were also degraded in HuH7 and THP-1 cells, 234 respectively, after treatment with 5-hydroxythalidomide (Fig. 4d and e). Endogenous IKZF1 235 showed no change in the presence of 5-hydroxythalidomide (Fig. 4e), even though PLZF was

236 degraded under the same conditions. Notably, dose-dependency between thalidomide and 5237 hydroxythalidomide in the biochemical CRBN-SALL4 interaction was almost identical 238 (middle panel in Fig. 4b). In addition, degradations of PLZF and SALL4 by 5239 hydroxythalidomide in cells occurred at almost the same levels as those in the thalidomide 240 treatment. Taken together with the function of human CYP3A ${ }^{25}$, these results suggest that 5241 hydroxythalidomide has potential similar to that of thalidomide for PLZF and SALL4 242 degradation in humans. 
Thalidomide-induced teratogenesis occurs in several animals, including zebrafish, 246 chicken, and rabbit ${ }^{4,10}$. The ZNF1/3 in PLZF (Fig3) and ZNF2 in SALL4 ${ }^{9,10}$ are important 247 regions for the interaction with CRBN. We thus compared these amino acid sequences among 248 vertebrates. The sequences of the ZNF1 and ZNF3 and other ZNFs in PLZF are significantly 249 conserved among many animals (Supplementary Fig. 6a), although the SALL4-ZNF2 250 sequence is not (Supplementary Fig. 6b). To investigate the thalidomide-dependent 251 degradation of Plzf or Sall4 by Crbn from other animals, the recombinant proteins of Crbn, 252 Plzf (Zbtb16 in chicken), and Sall4 from mouse (Mm) and chicken (Gg) were biochemically 253 analysed. In addition, Val388 of human CRBN is a key residue for thalidomide-dependent $254 \mathrm{CRBN}$-protein interaction ${ }^{7,9,10}$. Because the corresponding residue in mouse and chicken 255 Crbn is isoleucine (Supplementary Fig. 6c), we substituted the Ile to Val in both, to produce 256 MmCrbn-I391V and GgCrbn-I390V. In our biochemical analysis, the binding ability of 257 HsCRBN-V388I was dramatically decreased compared with that of the wild-type protein 258 (Supplementary Fig. 7a). Although MmCrbn did not bind with either protein in the presence 259 of thalidomide (middle panel), interestingly, wild-type GgCrbn bound to GgPlzf following 260 thalidomide treatment (right panel), whereas GgSall4 did not bind to GgCrbn with 261 thalidomide. Both MmCrbn-I391V and GgCrbn-I390V indicated highly thalidomide262 dependent binding with both Sall4 and Plzf.

264 did not provide an interaction between MmCrbn and MmPlzf. To investigate this reason, we 265 compared amino acid sequences of a thalidomide-binding region among human, mouse and 266 chicken (Supplementary Fig. 6c). As a result, Glu377 in HsCRBN was conserved in GgCrbn 267 but the Glu in MmCrbn was substituted to Vla. Actually, it was reported that Glu377 in 268 HsCRBN was an important amino acid for interaction between CRBN and GSPT1 ${ }^{8}$. 
269 Therefore, we investigated whether the Glu377 is important for the interaction between

270 CRBN and PLZF or SALL4. In vitro binding assay showed that substitution of the Glu to

271 Vla in HsCRBN significantly decreased binding ability to both SALL4 and PLZF

272 (Supplementary Fig. 7b). In MmCrbn, double substitution of the Vla380 to Glu and the Ile391

273 to Vla significantly increased binding ability to Sall4 and Plzf, although single substitution

274 of the Vla to Glu did not significantly increased (Supplementary Fig. 7c).

275 Next, each protein pair was transiently expressed in CRBN-deficient HEK293T cells.

276 In the mouse pair, wild-type MmCrbn did not degrade either MmPlzf or MmSall4, whereas

277 the MmCrbn-I391V and MmCrbn-V380E/I391V mutants degraded both (Fig. 5a and b,

278 respectively). In the chicken pair, wild-type GgCrbn significantly induced thalidomide-

279 dependent degradation of GgPlzf (Fig. 5c), while degradation of GgSall4 was almost never

280 observed (Fig. 5d). GgCrbn-I390V also degraded GgPlzf and GgSall4 with thalidomide (Fig.

$2815 \mathrm{c}$ and $\mathrm{d}$, respectively). In contrast, the GgCrbn-E379V mutant did not induce degradation of

282 GgPlzf (Supplementary Fig. 7d).

Taken together, we concluded that the Glu in thalidomide-binding region of CRBN is

284 an important amino acid for thalidomide-dependent interaction with PLZF and SALL4, and

285 that conservative amino acid sequence of CRBN-binding region with thalidomide in

286 substrate proteins was also required, like wild-type GgCrbn could not induce degradation of

287 GgSall4. In addition, these results suggest that thalidomide-dependent PLZF degradation 288 occurs in many animals, including chickens and humans, while in contrast, SALL4

289 degradation by thalidomide may occur in a limited number of animals, including rabbits ${ }^{10}$,

290 humans, and monkeys. 
291 Recently, humanized-CYP3A mice were reported to show abnormal limb development 292 after treatment with thalidomide ${ }^{25}$, suggesting that the thalidomide metabolites induce 293 teratogenesis. We therefore investigated the function of 5-hydroxythalidomide on mouse and 294 chicken Crbn-dependent degradation. Surprisingly, 5-hydroxythalidomide induced Crbn295 dependent degradation of both Plzf (Fig. 5e and g) and Sall4 (Fig. 5f and h), whereas 296 thalidomide had no function in MmCrbn and GgSall4 degradation by GgCrbn. Furthermore, 297 thalidomide and 5-hydroxythalidomide did not induce downregulation of PLZF and SALL4 298 mRNA expression in HuH7 cells (Supplementary Fig. 8). These results suggest that 5299 hydroxythalidomide, rather than thalidomide, has a high potential for degradation of both 300 Plzf and Sall4 in many animals.

\section{Plzf plays important roles in chicken limb development.}

It has been reported that both PLZF protein and Plzf mRNA are expressed in the limb buds of mouse and rat embryos ${ }^{14,33}$, suggesting direct function of Cbrn-thalidomide on PLZF in the developing limb bud. We thus investigated whether PLZF plays important roles in the development of chick limb bud. We first examined expression of Plzf gene in the chick limb

307 bud and confirmed Plzf mRNA expression by whole mount in situ hybridization (Fig. 6a).

308 Expression of Sall4 and Crbn genes was also observed in the same region (Fig.6a).

309 Expression of Plzf as well as Sall4 mRNA was confirmed to be in limb mesenchyme by 310 section in situ hybridization (Supplementary Fig. 9a). Then, to investigate whether 311 downregulation of Plzf mRNA induces limb teratogenicity in chicken embryos, we 312 constructed shRNA expression vector of GgPlzf. Immunoblot analysis showed that the 313 constructed shRNA vector downregulated protein expression of overexpressed GgPlzf in DF- 
3141 cells, which is chicken culture cells (Fig. 6b). Next, to elucidate developmental role of Plzf

315 in chick limb development, RCAN retrovirus, which express shRNA (\#2) against chick Plzf,

316 was infected into blastderm cells containing prospective lateral plate mesoderm cells that

317 gives rise to the limb bud. As shown Fig. 6c, it was confirmed that Plzf protein expression

318 level was downregulated in the chicken embryos treated with Plzf shRNA by immunoblot

319 analysis. In Fig. 6d, interestingly, Plzf shRNA infected limb bud showed several types of

320 malformations $(28 \%, \mathrm{n}=32)$. Forelimb and hindlimb were shortened compared to control

321 shRNA (GFP shRNA), infected limb bud $(0 \%, n=10)$. We also observed that only one bone

322 was formed in the zeugopod and digit number was also reduced in Plzf shRNA infected limb

323 bud. These results suggest that chick Plzf has a pivotal role for limb bud outgrowth and that

324 downregulation of Plzf causes teratogenicity.

\section{Thalidomide reduces Plzf protein levels in abnormal limb buds of chickens.}

Next, we investigated whether thalidomide targets PLZF in the chick limb bud. In previous reports, decreased expression of the $f g f 8$ gene is an indicator of abnormal limb bud

329 development following thalidomide treatment ${ }^{4}$, and we confirmed reduced/skewed pattern of $330 f g f 8$ in truncated limb buds after thalidomide treatment (Supplementary Fig. 10a). In 331 addition, limb skeletal defects were observed in this condition (Supplementary Fig. 10b). We 332 investigated whether the amount of Plzf protein changes in these abnormal limb buds. 333 Interestingly, reduced Plzf protein levels were observed in abnormal limb buds, although 334 immunostaining of the Sall4 protein showed no change (Fig. 7a). Furthermore, to confirm 335 the protein levels of Plzf and Sall4 in them, chick limb buds after thalidomide treatment were 336 collected and characterized. Immunoblotting analysis also indicated that a reduction in Plzf 
337 was predominantly observed in teratogenic limb buds (T3, T8, and T18 in Fig. 7b and c).

338 Taken together with the limb defects induced by human and mouse Plzf deficiency ${ }^{15,16}$, these

339 results suggest that PLZF is a pivotal molecule for teratogenesis in chicken. Furthermore, we

340 investigated whether 5-hydroxythalidomide induces teratogenicity in chicken embryo. As

341 showed in Fig. 7d, 5-hydroxythalidomide also induced teratogenicity, and immunoblot

342 analysis indicated that 5-hydroxythalidomide more strongly induced degradation of Plzf and

343 Sall4, compared to thalidomide (Fig. 7e). These results suggest that 5-hydroxythalidomide

344 plays an important role in thalidomide teratogenicity. The phenotypes of chicken embryos

345 provided between 5-hydroxythalidomide and thalidomide were very similar (Fig. 7d),

346 suggesting that degradation of other proteins including Sall4 is required much more to

347 provide severe teratogenicity.

\section{Discussion}

Many research groups have attempted to identify thalidomide-dependent substrates of

$351 \mathrm{CRBN}^{5-7,9,10,32}$. The methodologies they have used have largely been based on cell-based assays such as SILAC (stable isotope labelling by amino acids in cell culture) and transient

353 expression systems. In this study, we have used a biochemical assay using cell-free HuTFPA

354 and AlphaScreen technologies. This method could easily detect thalidomide-dependent

355 interactions between CRBN and PLZF, SALL4, or IKZF1, without the need for protein

356 purification (Fig. 1), as well as the variations in the biochemical response of CRBN-protein

357 depending on the presence of thalidomide, its derivatives, or 5-hydroxythalidomide (Fig. 3

358 to 5). Thalidomide-dependent interaction between CRBN and PLZF related to two ZNF

359 domains (Fig. 3) and slowness of IMiD-dependent protein degradation, suggesting that the 
360 interaction manner is more complicated manner and may be different from known substrates, 361 such as IKZF1, IKZF3, and SALL4. These results support a reason that PLZF has not been 362 identified by method using cell-based assay or in silico so far, suggesting that our new 363 biochemical approach using protein array could be useful in the identification. However, our 364 assay system cannot identify substrate which requires the interaction with CRBN in protein 365 complex. Therefore, our cell-free and conventional cell-based assay are complementary 366 relationship each other, and we believe that the cell-free method can be used in the future 367 research for exploration and confirmation of substrates with the cell-based assay. 368 Furthermore, as IKZF1 interacted with CRBN in the presence of thalidomide but not 5369 hdroxythalidomide, this method would be used for analysis of compound-dependent protein370 protein interaction in development of novel drugs including thalidomide derivatives.

In this study, we identified PLZF as a new target of the thalidomide-CRBN system. In 372 chicken embryo, downregulation of Plzf showed hypoplasia of limb bud (Fig. 6d and e), 373 indicating that Plzf is required for proper chicken limb development. Furthermore, 374 thalidomide and 5-hydroxythalidomide treatments decreased Plzf protein level but Sall4 was 375 not induced protein degradation in the abnormal limb buds of chicken embryos (Fig. 7). 376 According to these findings, we made a model for teratogenesis of chick limb buds (Fig. 8a). 377 In mouse studies, $\mathrm{Plzf}^{-/}$deficient mice had major musculoskeletal limb defects ${ }^{14}$, and it was 378 reported that PLZF deficient caused alteration of Hoxds or Bmps expression in developing $379 \mathrm{limb}^{14}$. Because Bmp proteins function as regulators in programming cell death and Plzf/Gli3 380 deficient induced cell death ${ }^{15}$, suggesting that degradation of PLZF by thalidomide treatment 381 may affect cell proliferation in limb development. In contrast, Sall4 conditional knockout 382 mice (Sall4-CKO) driven by T-Cre, which express early mesoderm, did not show any 383 phenotype in the forelimb $b^{12}$. Furthermore, only $5 \%$ of $\mathrm{Plzf}^{-/-}$mice showed forelimb phenotype 
384 which shows autopod abnormality ${ }^{14}$. These results indicate that thalidomide-induced

385 teratogenicity in human cannot be explained by the results of each single knockout mice of

386 Sall4 or Plzf. In previous report, Plzf and Gli3 double knockout mice showed phenotypes of 387 remarkably reduction of stylopod and zeugopod ${ }^{15}$, and these phenotypes similar to 388 phocomelia that is a typical phenotype of thalidomide embryopathy. It was reported that Gli3 389 expression was reduced in Sall4-CKO mice, therefore, we expect that double knockout mice 390 of Sall4; T-Cre; Plzf ${ }^{-/}$would explain thalidomide phenotype in human patients. Our chick 391 results showed that PLZF would be the pivotal target of thalidomide. Given that Sall4 protein 392 was not degraded by thalidomide treatment in the chick embryo, it is thought that PLZF has 393 more crucial function for normal development of the limb than Plzf in the mouse. Thus, 394 variation of the protein sensitivity to the thalidomide and/or difference of the genes that are 395 necessary for normal development of the limb between species would bring about difference 396 of the phenotype between species in thalidomide teratology, which we showed in this study. 397 PLZF that we found in this study as a new target of thalidomide will be a crucial target to 398 solve thalidomide mystery between species in future.

PLZF is expressed in many cell types ${ }^{34}$, and is a multifunctional TF modulating many 400 developmental biological processes ${ }^{13}$ including cellular proliferation and cell cycle control, 401 myeloid and lymphoid cell development and differentiation, programming of NKT and iNKT 402 cells, spermatogenesis and spermatogonial stem cell renewal, haematopoiesis, 403 musculoskeletal-limb development, megakaryocytic development, and cytokine production. 404 Notably, PLZF functions as a regulator for many immunoresponses ${ }^{35-38}$. Thalidomide is an 405 immunomodulatory imide drug, and IKZF1 and IKZF3 are thought to be key targets of 406 immunomodulation by thalidomide and its derivatives ${ }^{39,40}$. Therefore, dysfunction of PLZF 
407 by thalidomide may also result from its function as an IMiD, though further analysis will be 408 required to confirm this.

Human CYP2C and 3A produce two metabolites: 5-hydroxythalidomide and 5'410 hydroxythalidomide, as primary metabolites of thalidomide ${ }^{18-20} .5^{\prime}$-hydroxythalidomide has 411 no effect on teratogenesis in chicken embryos ${ }^{23}$. However, it was no evidence whether 412 metabolites from thalidomide induce CRBN-dependent protein degradation. In this study, 5413 hydroxythalidomide induced degradation of both human PLZF and SALL4 proteins at the 414 same level as thalidomide (Fig. 4). It is known that humans are highly sensitive to 415 thalidomide ${ }^{3,41}$. These reports and our results suggest that 5-hydroxythalidomide has the 416 potential for teratogenesis. For this reason, we used our findings to develop the hypothesis 417 that the double degradations of PLZF and SALL4 by both thalidomide and 5418 hydroxythalidomide produce high sensitivity to thalidomide in human embryopathy (Fig. 8b).

419 We are convinced that researching the generation and action of 5-hydroxythalidomide will 420 be important for understanding the function of thalidomide.

421 Thalidomide is a typical drug which shows species specificity ${ }^{3}$. From several 422 researches, the species specificity of thalidomide has been thought to be provide by the 423 difference of a thalidomide-binding sequence in $\mathrm{CRBN}^{7,9,10}$. Consistent with these reports, in 424 this study, humanized-mouse and chicken Crbns indicated thalidomide-dependently 425 interactions and degradation of both Plzf and Sall4 proteins (Fig. 5a-d and Supplementary 426 Fig. 7). In addition, with the sequence difference in Crbn, it has been thought that the 427 metabolism cascade of thalidomide is also important in the species specificity ${ }^{3}$. In this study, 428 5-hydroxythalidomide showed higher potential than thalidomide for degradations of both 429 Plzf and Sall4 proteins from mouse and chicken (Fig. 5e-h, Fig. 7d and e). Furthermore, 
430 humanized-CYP3A mice have been recently shown to undergo abnormal limb development

431 at a high rate $(>40 \%)$ after thalidomide treatment ${ }^{25}$, although in general thalidomide does not 432 produce teratogenesis in mice ${ }^{3}$. These reports and our results suggest that thalidomide 433 metabolism cascade, including metabolism speed and kinds of metabolites, plays an 434 important role in the species specificity of thalidomide. 
435

436

437

438

439

440

441

442

443

444

445

446

447

448

449

450

451

452

453

454

455

456

457

458

459

460

461

462

463

464

465

466

\section{Methods}

Reagents. Thalidomide (Sigma-Aldrich and Tokyo Chemical Industry Co., Ltd), Pomalidomide (Sigma-Aldrich), Lenalidomide (FUJIFILM Wako Pure Chemical), 5hydroxythalidomide (5-hydroxythalidomide was prepared according to a previously published method $^{20}$ ), MG132 (Peptide Institute), and MLN4924 (Chemscene) were dissolved in DMSO (FUJIFILM Wako Pure Chemical) at 2 to $100 \mathrm{mM}$ and stored at $-20^{\circ} \mathrm{C}$ as stock solutions. All drugs were diluted 1,000, 500, or 250-fold for in vivo experiments, or diluted 200-fold for in vitro experiments.

Production of recombinant proteins using the cell-free system. In vitro transcription and wheat cell-free protein synthesis were performed using a WEPRO1240 expression kit (CellFree Sciences). Transcripts were conducted using SP6 RNA polymerase with plasmids or DNA fragments as templates. The translation reaction was performed in bilayer mode using a WEPRO1240 expression kit (Cell-Free Sciences), according to the manufacturer's instructions. For biotin labelling, $1 \mu \mathrm{l}$ of cell-free synthesized crude biotin ligase (BirA), produced by the wheat cell-free expression system, was added to the lower layer, and $0.5 \mu \mathrm{M}$ (final concentration) of d-biotin (Nacalai Tesque) was added to both the upper and lower layers, as described previously ${ }^{42}$.

Interaction analysis of CRBN-IMiD-substrate using AlphaScreen technology. IMiD at the concentrations indicated in each figure and $0.5 \mu 1$ of biotinylated HsCRBN, MmCrbn, or

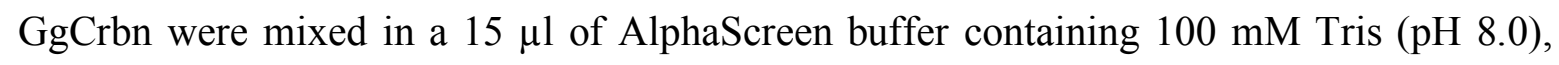
$0.01 \%$ Tween 20, $100 \mathrm{mM} \mathrm{NaCl}$, and $1 \mathrm{mg} / \mathrm{ml}$ BSA. Then, $5 \mu \mathrm{l}$ of substrate mixture containing $0.8 \mu \mathrm{l}$ of FLAG-GST-substrate in AlphaScreen buffer was added, and $20 \mu \mathrm{l}$ of the reaction mixture was incubated at $26^{\circ} \mathrm{C}$ for $1 \mathrm{~h}$ in a 384 -well AlphaPlate (PerkinElmer). Subsequently, $5 \mu$ l of detection mixture containing $0.2 \mu \mathrm{g} / \mathrm{ml}$ anti-DYKDDDDK mouse mAb (Wako), $0.08 \mu \mathrm{l}$ of streptavidin-coated donor beads, and $0.08 \mu \mathrm{l}$ of Protein A-coated acceptor beads (PerkinElmer) in AlphaScreen buffer, were added to each well. After incubation at $26^{\circ} \mathrm{C}$ for $1 \mathrm{~h}$, luminescent signals were detected using an EnVision plate reader (PerkinElmer). 
Production of the human transcription factor protein array (HuTFPA). For the construction of human TF protein array, we prepared pEU-E01-FLAG-GST-K1-02 vector containing FLAG tag, GST tag, SG linker, and AsiSI restriction enzyme site at 5' upstream of multiple cloning site. cDNA clones coding proteins with DNA-binding domains were selected from cDNA resources collected by Kazusa DNA research institute ${ }^{43}$ (Supplementary Table 1). The plasmid of each clone was digested by combination of AsiSI and an appropriate restriction enzyme such as XhoI, SalI or NotI. The DNA fragment was inserted into pEUE01-FLAG-GST-K1-02 vector digested by same restriction enzymes. After subcloning, pEU expression plasmids were arranged in 96 well format and stored as glycerol stock. Transcription template DNA fragments were amplified directly by PCR using PrimeStar Max PCR polymerase (Takara Bio), SPu-2 (5'-CAGTAAGCCAGATGCTACAC) and AODA2306 (5'-AGCGTCAGACCCCGTAGAAA) primers and diluted glycerol stocks as template. Transcription and translation reactions were conducted using WEPRO7240 expression kit (Cell-Free Sciences) in micro-titer plate format. Transcription reaction mixture was prepared by mixing $1.4 \mu \mathrm{l}$ of transcription buffer LM, $0.7 \mu 1$ of NTP mixture ( $25 \mathrm{mM}$ each), $0.07 \mu \mathrm{l}$ RNase Inhibitor (Promega), $0.26 \mu \mathrm{l} \mathrm{SP6} \mathrm{polymerase} \mathrm{(Promega)} \mathrm{and} 1.4 \mu \mathrm{l}$ PCR product in 96 well plate. The transcription reaction was incubated at $37^{\circ} \mathrm{C}$ for $18 \mathrm{~h}$. Translation reaction mixture containing $2.5 \mu \mathrm{l}$ of mRNA, $1.67 \mu \mathrm{l}$ of WEPRO 7240 wheat germ extract, $0.14 \mu \mathrm{l}$ of creatine kinase $(20 \mathrm{mg} / \mathrm{ml})$ (Roche diagnostics) and $0.11 \mu 1 \mathrm{RNase}$ Inhibitor was prepared and overlaid with $44 \mu 1$ of SUB-AMIX SGC solution (Cell-Free Sciences) in V-bottom 384 well plate. The translation reaction was incubated at $26^{\circ} \mathrm{C}$ for 18 $\mathrm{h}$. Expression of each protein product was confirmed by Western blotting using antiDYKDDDDK tag antibody (FUJIFILM Wako Pure Chemical).

493 buffer, to 384-well AlphaPlates using a FlexDrop Precision Reagent Dispenser 494 (PerkinElmer). We next added $0.8 \mu 1$ of FLAG-GST-transcription factor proteins to 384-well AlphaPlates using a NanoHead (PerkinElmer) and a Janus Workstation (PerkinElmer). After the 384-well AlphaPlates were incubated at $26^{\circ} \mathrm{C}$ for $1 \mathrm{~h}, 5 \mu \mathrm{l}$ of detection mixture containing $4970.2 \mu \mathrm{g} / \mathrm{ml}$ anti-DYKDDDDK mouse mAb (FUJIFILM Wako Pure Chemical), $0.08 \mu l$ of 498 streptavidin-coated donor beads, and $0.08 \mu \mathrm{l}$ of Protein A-coated acceptor beads 499 (PerkinElmer) in AlphaScreen buffer, were added to each well using a FlexDrop precision 
500

501

502

503

504

505

506

507

508

509

510

511

512

513

514

515

516

517

518

519

520

521

522

523

524

525

526

527

528

529

530

531

532

reagent dispenser. After incubation at $26^{\circ} \mathrm{C}$ for $1 \mathrm{~h}$, luminescent signals were detected using an EnVision plate reader (PerkinElmer).

Plasmids. Plasmids pDONR221 and pcDNA3.1(+), based on Gateway technology, were purchased from Invitrogen, and the pEU vector for the wheat cell-free system was constructed in our laboratory, as previously described ${ }^{26}$. Plasmids pcDNA3.1(+)-FLAG-GW, pcDNA3.1(+)-FLAG-MCS, pcDNA3.1(+)-AGIA-MCS, pEU-bls-GW, and pEU-bls-MCS were constructed based on each original vector by PCR and using the In-Fusion system (Takara Bio), or PCR and restriction enzymes. pEU-FLAG-GST-IKZF1, -SALL4, -PLZF, SALL1, -SALL2, -ZBTB17, -ZBTB20, -ZBTB38, -ZBTB48, -HNRNPK, -HMGB2, and ELF5 were purchased from the Kazusa DNA Research Institute. Plasmids HsSALL4, HsPLZF, and IKZF1 were amplified and restriction enzyme sites were added by PCR and cloned into pcDNA3.1(+)-AGIA-MCS. The open reading frame of HsCRBN was purchased from the Mammalian Gene Collection (MGC) and MmCrbn, MmSall4, and MmPlzf were did from Functional Annotation of Mouse (FAMTOM), respectively ${ }^{28,31}$. The open reading frame of GgCrbn was artificially synthesized by IDT, and pcDNA3.1(+)-GgSall4DYKDDDDK and pcDNA3.1(+)-GgPlzf-DYKDDDDK were purchased from GenScript. HsCRBN was amplified and the BP reaction sequence (attB and attP) was added by PCR and cloned into pDONR221 using BP recombination (Invitrogen). Then, pDONR221-HsCRBN was recombined to pEU-bls-GW or pcDNA3.1(+)-FLAG-GW using LR recombination (attL and attR). MmCrbn and GgCrbn were amplified and restriction enzyme sites were added by PCR and cloned into pEU-bls-MCS or pcDNA3.1(+)-FLAG-MCS. MmSall4, GgSall4, MmPlzf, and GgPlzf were amplified and restriction enzyme sites were added by PCR and cloned into pEU-FLAG-GST-MCS or pcDNA3.1(+)-AGIA-MCS. Domain swapped HsPLZF was constructed by inverse PCR and the In-Fusion system (Takara Bio). Deletion mutation and amino acid mutation of each protein was performed by inverse PCR.

For in situ hybridization, the cDNAs for chicken Fgf8, Crbn, Sall4, and Plzf were obtained by PCR using the following primers: Fgf8 (NM_001012767.1), 5' attacgcgtATGGACCCCTGCTCCTCGCTCTTCA-3' and 5' attgataTCATGGGCGCAGGGAGGCGCTGGAG-3'; Crbn (XM_015293204.2), 5' ctataggctagaattcacgcgtATGGCCGCCGAGGAGGGAGGTGACGGA-3' and 5' cactaaagggaagcggecgcgatatcTTACAAGCAGAGTAACGGAGATC-3'; $\quad$ Sall4 (NM_001080872.1), 
and

$5^{\prime}-$ cactaaagggaagcggecgegataTCAGACGTAGCAGAGGTAGAGATAG-3'. The amplified fragments of $F g f 8$ were digested by MluI-EcoRV, and subcloned into pCMS-EGFP vector (Clontech). The amplified fragments of Crbn, Sall4, and Plzf were inserted into MluI-EcoRV site of pCMS-EGFP vector by In-fusion (Takara Bio).

For knockdown of chick Plzf, the shRNA sequences of chick Plzf (\#1: 5' GGAAATCGAGGTACATCAAGG-3' or \#2: 5'-GATTACTCGGCCATGATCAAA-3') were used. The following

DNA

oligos:

$\# 1:$

$5^{\prime}-$ gatcccGGAAATCGAGGTACATCAAGGgcttcctgtcacCCTTGATGTACCTCGATTTCCttt ttta-3' and 5'-agcttaaaaaaGGAAATCGAGGTACATCAAGGgtgacaggaagc CCTTGATGTACCTCGATTTCCgg-3'; \#2: gatcccGATTACTCGGCCATGATCAAAgcttcctgtcacTTTGATCATGGCCGAGTAATCttt ttta-3' and 5'-agcttaaaaaaGATTACTCGGCCATGATCAAAgtgacaggaagc TTTGATCATGGCCGAGTAATgg-3' were purchased from Invitrogen. The DNA oligo pairs were annealed and inserted into pEntryCla12-chickU6 shuttle vector using BamHI/HindIII site.

Cell culture and transfection. HEK293T cells were cultured in DMEM (low glucose) medium (FUJIFILM Wako Pure Chemical) supplemented with 10\% fetal bovine serum (FUJIFILM Wako Pure Chemical), $100 \mathrm{unit} / \mathrm{ml}$ penicillin, and $100 \mu \mathrm{g} / \mathrm{ml}$ streptomycin (Gibco) at $37^{\circ} \mathrm{C}$ under 5\% $\mathrm{CO}_{2}$. HEK293T cells were transfected using TransIT-LT1 transfection reagent (Mirus Bio) or PEI Max: Polyethyleneimine "Max" (MW 40,000) (PolyScience, Inc.).

HuH7 cells were cultured in DMEM (high glucose) medium (FUJIFILM Wako Pure $561 \mu \mathrm{g} / \mathrm{ml}$ streptomycin (Gibco), $1 \mathrm{mM}$ Sodium Pyruvate (Gibco), $10 \mathrm{mM}$ HEPES (Gibco), and $1 \times$ MEM NEAA (Gibco) at $37^{\circ} \mathrm{C}$ under $5 \% \mathrm{CO}_{2}$.

THP-1 cells were cultured in RPMI160 GlutaMAX medium (Gibco) supplemented with

$56410 \%$ fetal bovine serum (FUJIFILM Wako Pure Chemical), 100 unit $/ \mathrm{ml}$ penicillin, and 100 $565 \mu \mathrm{g} / \mathrm{ml}$ streptomycin (Gibco) at $37^{\circ} \mathrm{C}$ under $5 \% \mathrm{CO}_{2}$. 
566

TK, HT, BJAB, SU-DHL-4, MT-4, Raji cells were cultured in RPMI1640 GlutaMAX medium supplemented with 10\% fetal bovine serum (FUJIFILM Wako Pure Chemical), 100 unit/ml penicillin, $100 \mu \mathrm{g} / \mathrm{ml}$ streptomycin (Gibco), and $55 \mu \mathrm{M}$ 2-Mercaptoethanol (Gubco) at $37^{\circ} \mathrm{C}$ under $5 \% \mathrm{CO}_{2}$.

DF-1 cells were cultured in DMEM (low glucose) medium (FUJIFILM Wako Pure Chemical) supplemented with 10\% fetal bovine serum (Wako), 100 unit/ml penicillin, and $100 \mu \mathrm{g} / \mathrm{ml}$ streptomycin (Gibco) at $37^{\circ} \mathrm{C}$ under $5 \% \mathrm{CO}_{2}$. DF-1 cells were transfected using TransIT-LT1 transfection reagent (Mirus Bio).

Immunoblot and antibodies. Protein lysates were separated by SDS-PAGE and transferred onto polyvinylidene difluoride membranes (Millipore). After the membranes were blocked using 5\% skimmed milk (Megmilk Snow Brand) in TBST (20 mM Tris-HCl pH 7.5, 150 $\mathrm{mM} \mathrm{NaCl}, 0.05 \%$ Tween 20 ) at room temperature for $1 \mathrm{~h}$, the following antibodies were used. Anti-FLAG mouse mAb (HRP-conjugated, Sigma-Aldrich, A8592), anti-AGIA rabbit $\mathrm{mAb}^{31}$ (HRP-conjugated, produced in our laboratory) were used to detect epitope-tagged proteins. Anti- $\alpha$-tubulin rabbit pAb (HRP-conjugated, MBL, PM054-7) was used to detect $\alpha$-tubulin. Biotinylated proteins were detected by anti-biotin (HRP-conjugated, Cell Signaling Technology, \#7075). Anti-CRBN rabbit mAb (Cell Signaling Technology, \#71810), anti-PLZF rabbit mAb (Cell Signaling Technology, \#39784), anti-PLZF rabbit pAb (GeneTex, GTX111046), anti-SALL4 rabbit pAb (Abcam, ab29112), anti-SALL4 mouse mAb (Santa Cruz Biotechnology, sc-101147), anti-DDB1 mouse mAb (Santa Cruz Biotechnology, sc-376860), anti-CUL4 mouse mAb (Santa Cruz Biotechnology, sc-377188), anti-RBX1 mouse mAb (Santa Cruz Biotechnology, sc-393640), and anti-ubiquitin mouse mAb (P4D1, Cell Signaling Technology, \#3936) were used as primary antibodies. Antirabbit IgG (HRP-conjugated, Cell Signaling Technology, \# 7074) and anti-mouse IgG (HRPconjugated, Cell Signaling Technology, \# 7076) were used as secondary antibodies. Immobilon (Millipore) or ImmunoStar LD (FUJIFILM Wako Pure Chemical) was used as substrate HRP and luminescent signals were detected using an ImageQuant LAS 4000mini (GE Healthcare). To perform re-probing, Stripping Solution (FUJIFILM Wako Pure Chemical) was used and re-blocked using 5\% slim milk in TBST.

For immunoblot analysis of extract from chicken embryo, a right forelimb bud was dissected from $\mathrm{HH}$ st. 22/23 embryos, and boiled in $50 \mu \mathrm{l}$ of buffer (50 mM Tris-HCl pH 7.5, 
598

599

600

601

602

603

604

605

606

607

608

609

610

611

612

613

614

615

616

617

618

619

620

621

622

623

624

625

626

627

628

629

$4 \%$ SDS) at $98^{\circ} \mathrm{C}$ for $10 \mathrm{~min}$. Protein concentration of each lysate was quantified using BCA assay (Thermo Fisher Scientific).

In vitro pull-down assay of CRBN and substrate. To confirm the thalidomide-dependent interactions between IKZF1, PLZF, or SALL4 and CRBN, we performed pull-down assays using Dynabeads M-280 Streptavidin (Invitrogen). Biotinylated CRBN-WT and CRBNYW/AA were synthesized using the wheat cell-free system as described above. We then mixed $5 \mu$ l of Dynabeads M-280 Streptavidin with $5 \mu 1$ of biotinylated CRBN-WT or CRBNYW/AA and diluted this 10-fold with PBS containing 0.05\% Tween20, and incubated this at room temperature for $1 \mathrm{~h}$. The beads were washed three times in $500 \mu \mathrm{l}$ PBS containing $0.05 \%$ Tween 20 and substrate-thalidomide mixture was added containing $10 \mu 1$ of FLAGGST-IKZF1, -SALL4 or -PLZF, and $200 \mu \mathrm{M}$ thalidomide $(0.5 \%$ DMSO) in $300 \mu$ of AlphaScreen buffer containing $100 \mathrm{mM} \mathrm{NaCl}, 0.01 \%$ Tween20, and $1 \mathrm{mg} / \mathrm{ml}$ BSA. After rotation at room temperature for $90 \mathrm{~min}$, the beads were washed four times in $500 \mu \mathrm{l}$ of $1 \times$ Lysis buffer (50 mM Tris-HCl pH 7.5, $150 \mathrm{mM} \mathrm{NaCl,} \mathrm{1 \%} \mathrm{Triton} \mathrm{X-100)} \mathrm{and} \mathrm{proteins} \mathrm{were}$ eluted by boiling in $1 \times$ sample buffer $(62.5 \mathrm{mM}$ Tris- $\mathrm{HCl} \mathrm{pH} 6.8,2 \%$ SDS, $10 \%$ glycerol) containing 5\% 2-mercaptoethanol. The proteins were then analysed by immunoblot.

Construction of CRBN-KO HEK293T cells. The guide nucleotide sequence 5'ACTCCGGGCGGTTACCAGGC- 3' was selected from the human CRBN gene. The Guideit plasmid vector (Takara Bio) was used to construct CRBN-KO cells. We then cultured HEK293T cells in a 6-well plate and transfected the plasmid into them. Two days after transfection, GFP positive cells were sorted by FACSAria (Becton, Dickinson and Company) and cell clones were obtained by limiting dilution. Genomic DNA was then isolated and the mutation was confirmed by sequencing after TA cloning (Toyobo).

In vivo IMiD-dependent degradation assay of substrates. To confirm IMiD-dependent degradation of PLZF, HEK293T or HEK293T-CRBN ${ }^{-/-}$cells were cultured in 48-well plates and transfected with $200 \mathrm{ng}$ pcDNA3.1(+)-FLAG-CRBN-WT or $200 \mathrm{ng}$ pcDNA3.1(+)FLAG-CRBN-YW/AA and $20 \mathrm{ng}$ pcDNA3.1(+)-AGIA-PLZF or -AGIA-PLZF variants, or -AGIA-SALL4. After the cells were transfected for $8 \mathrm{~h}$, they were treated with IMiD or DMSO $(0.1 \%)$ in culture medium, at the times and concentrations indicated in each figure. 
630

631

632

633

634

635

636

637

638

639

640

641

642

643

644

645

646

647

648

649

650

651

652

653

654

655

656

657

658

659

660

661

To show that IMiD-dependent PLZF degradation is caused by CRL and the 26S proteasome, the cells were treated with $2 \mu \mathrm{M}$ MLN4924 and $10 \mu \mathrm{M}$ MG132 (0.2\% DMSO) at the times indicated in Fig. 2a.

To examine the degradation of endogenous PLZF or SALL4, we cultured HEK293T, HuH7, or THP-1 cells in 24 or 48-well plates and treated them with lenalidomide or DMSO $(0.1 \%)$ in culture medium at the times and concentrations indicated in each figure.

To examine the degradation of endogenous PLZF in TK, HT, BJAB, SU-DHL-4, MT-4, Raji cells, we cultured induced the lymphoma cells in 12-well plate and treated then with lenalidomide, pomalidomide or DMSO $(0.1 \%)$ in culture medium at the times and concentrations indicated in Supplementary Fig.5. Then, the cells were lysed with XXX $\mu \mathrm{L}$ of RIPA buffer containing a protease inhibitor cocktail (Sigma-Aldrich). Protein concentration of each lysate was quantified using BCA assay (Thermo Fisher Scientific).

To examine 5-hydroxythalidomide-dependent degradation of overexpressed PLZF, SALL4 or IKZF1, HEK293T-CRBN ${ }^{-/}$cells were cultured in 48-well plates and transfected with $200 \mathrm{ng}$ pcDNA3.1(+)-FLAG-CRBN-WT and 20 ng pcDNA3.1(+)-AGIA-SALL4, PLZF, or -IKZF1. After the cells were transfected for $8 \mathrm{~h}$, they were treated with thalidomide, 5-hydroxythalidomide, or DMSO $(0.1 \%)$ in culture medium at the indicated times and concentrations in Fig. 4c. For endogenous SALL4, PLZF, and IKZF1, HuH7 or THP-1 cells were cultured in 48-well plates and treated with thalidomide, 5-hydroxythalidomide, or DMSO $(0.1 \%)$ in culture medium at the indicated times and concentrations in Fig. $4 \mathrm{~d}$ and $4 \mathrm{e}$.

To examine the species specificity of IMiD-dependent protein degradation, HEK293T$\mathrm{CRBN}^{-/}$cells were cultured in 48-well plates and transfected with $200 \mathrm{ng}$ pcDNA3.1(+)FLAG-(mouse or chicken) Crbn-WT or -IV and $20 \mathrm{ng}$ pcDNA3.1(+)-AGIA-(mouse or chicken) Plzf or -(mouse or chicken) Sall4. After the cells were transfected for $8 \mathrm{~h}$, they were treated with thalidomide or DMSO $(0.2 \%)$ in culture medium for the times and concentrations indicated in Fig. 5a-d.

To examine whether 5-hydroxythalidomide induced the degradation of (mouse or chicken) Sall4 or Plzf, HEK293T-CRBN-/- cells were cultured in 48-well plates and transfected with $200 \mathrm{ng}$ pcDNA3.1(+)-FLAG-(mouse or chicken) Crbn-WT and $20 \mathrm{ng}$ pcDNA3.1(+)-AGIA-(mouse or chicken) Sall4 or -(mouse or chicken) Plzf. After the cells were transfected for $8 \mathrm{~h}$, they were treated with thalidomide, 5-hydroxythalidomide or DMSO $(0.2 \%)$ in culture medium for the times and concentrations indicated in Fig. 5e-h. 
662

663

664

665

666

667

668

669

670

671

672

673

674

675

676

677

678

679

680

681

682

683

684

685

686

687

688

689

690

691

692

693

In all experiments, cells were lysed by boiling in $1 \times$ sample buffer containing $5 \% 2$ mercaptoethanol, and the lysates were analysed by immunoblot.

Quantitative RT-PCR. To demonstrate PLZF protein level decrease results from posttranslational event, PLZF mRNA expression in HEK293T, HuH7, or THP-1 cells treated with DMSO or lenalidomide for $24 \mathrm{~h}$, were assessed by quantitative Real-Time PCR (qRTPCR). To analyse SALL4 or PLZF mRNA expression, HuH7 cells were treated with DMSO, thalidomide or 5-hydroxythalidomide for $24 \mathrm{~h}$, were assessed by qRT-PCR. Total RNA was isolated from HEK293T, HuH7, or THP-1 cells treated with DMSO or lenalidomide for 24 h using a SuperPrep cell lysis kit (Toyobo) and cDNA was synthesized using a SuperPrep RT kit (Toyobo), according to the manufacture's protocol. RT-PCR was performed using a KOD SYBR qPCR Mix (Toyobo) and data was normalized against GAPDH mRNA levels. PCR primers are as follows: PLZF FW 5'-GCACAGTTTTCGAAGGAGGA-3', PLZF RV 5'-GGCCATGTCAGTGCCAGT-3'，SALL4 FW 5'-GGTCCTCGAGCAGATCTTGT-3', SALL4 RV 5'-GGCATCCAGAGACAGACCTT-3', GAPDH FW 5'AGCAACAGGGTGGTGGAC-3', GAPDH RV 5'-GTGTGGTGGGGGACTGAG-3'

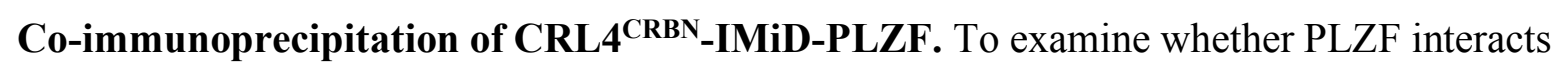
with CRL4 ${ }^{\text {CRBN }}$-thalidomide or -lenalidomide, HEK293T cells were cultured in a $15-\mathrm{cm}$ dish and transfected with $12 \mu \mathrm{g}$ pcDNA3.1(+)-FLAG-CRBN and $12 \mu \mathrm{g}$ pcDNA3.1(+)-AGIAPLZF. After HEK293T cells were treated with DMSO, $10 \mu \mathrm{M}$ or $100 \mu \mathrm{M}$ thalidomide or lenalidomide in the presence of $10 \mu \mathrm{M}$ MG132 for $8 \mathrm{~h}$, cells were lysed in $1.5 \mathrm{~mL}$ IP Lysis buffer (Pierce) (25 mM Tris-HCl pH 7.5, $150 \mathrm{mM} \mathrm{NaCl,} 1 \mathrm{mM}$ EDTA, 1\% NP-40, 5\% glycerol) containing a protease inhibitor cocktail (Sigma-Aldrich) and incubated on ice for $15 \mathrm{~min}$. Lysates were clarified by centrifugation at $13,000 \mathrm{rpm}$ for $15 \mathrm{~min}$, and $\mathrm{CRL}^{\mathrm{CRBN}}$ was immunoprecipitated using anti-FLAG M2 magnetic beads (Sigma-Aldrich) with DMSO or $10 \mu \mathrm{M}$ or $100 \mu \mathrm{M}$ thalidomide or lenalidomide in the presence of $10 \mu \mathrm{M}$ MG132. After overnight rotation at $4^{\circ} \mathrm{C}$, the beads were washed three times with $800 \mu \mathrm{L}$ of IP Lysis buffer (Pierce) and the proteins were eluted with $1 \times$ sample buffer. After eluted proteins were transferred to another tube, 2-mercaptoethanol (final concentration is 5\%) was added, and boiled at $98^{\circ} \mathrm{C}$ for $5 \mathrm{~min}$. The proteins were then analysed by immunoblot. 
694 In vivo ubiquitination assay. To detect polyubiquitination of PLZF in cells, HEK293T cells

695

696

697

698

699

700

701

702

703

704

705

706

707

708

709

710

711

712

713

714

715

716

717

718

719

720

721

722

723

724

725

726

were cultured in a 10-cm dish and transfected with $5 \mu \mathrm{g}$ pcDNA3.1(+)-FLAG-CRBN and 5 $\mu \mathrm{g}$ pcDNA3.1(+)-AGIA-PLZF. After HEK293T cells were treated with DMSO, $10 \mu \mathrm{M}$ or $100 \mu \mathrm{M}$ thalidomide or lenalidomide in the presence of $10 \mu \mathrm{M}$ MG132 for $10 \mathrm{~h}$, cells were lysed in $600 \mu \mathrm{l}$ of SDS Lysis buffer (50 mM Tris-HCl pH 7.5, 1\% SDS) containing a protease inhibitor cocktail (Sigma-Aldrich) and boiled at $90^{\circ} \mathrm{C}$ for $15 \mathrm{~min}$. Denatured lysates were treated with Benzonase Nuclease (Sigma-Aldrich) at $37^{\circ} \mathrm{C}$ for $1 \mathrm{~h}$, and the lysates were clarified by centrifugation at 13,000 rpm for $15 \mathrm{~min}$, then diluted 10-fold with IP Lysis buffer (Pierce). The proteins were immunoprecipitated overnight with Dynabeads Protein G (Invitrogen) interacting anti-AGIA antibody at $4^{\circ} \mathrm{C}$, which were then washed three times with $800 \mu 1$ of IP Lysis buffer (Pierce). Proteins were eluted by boiling in $1 \times$ sample buffer containing 5\% 2-mercaptoethanol. The proteins were then analysed by immunoblot.

In vitro binding and ubiquitination assay. To confirm that PLZF is a direct substrate, we performed an in vitro binding and ubiquitination assay as described previously ${ }^{6}$. HEK293T cells were cultured in a $15-\mathrm{cm}$ dish and transfected with $28 \mu \mathrm{g}$ pcDNA3.1(+)-FLAG-CRBN or empty vector. HEK293T cells were cultured in two $15-\mathrm{cm}$ dishes and transfected with 25 $\mu \mathrm{g}$ pcDNA3.1(+)-AGIA-PLZF per one dish. After $24 \mathrm{~h}$, the cells were lysed in $1.6 \mathrm{ml} / \mathrm{dish}$ of IP Lysis buffer (Pierce) containing a protease inhibitor cocktail (Sigma-Aldrich), and 400 $\mu 1$ of FLAG-CRBN or empty vector lysates were mixed with $400 \mu \mathrm{L}$ of AGIA-PLZF lysates in the presence of DMSO or $100 \mu \mathrm{M}$ lenalidomide. The FLAG-CRBN and AGIA-PLZF mixtures were immunoprecipitated using anti-FLAG M2 magnetic beads by rotating overnight at $4{ }^{\circ} \mathrm{C}$. As a negative control, empty vector and AGIA-PLZF mixtures were immunoprecipitated using anti-AGIA-conjugated magnetic beads (produced in our laboratory) by rotating overnight at $4^{\circ} \mathrm{C}$. The beads were washed three times with $800 \mu 1$ of IP Lysis buffer (Pierce) and washed twice with $600 \mu \mathrm{l}$ of $1 \times$ ubiquitin reaction buffer (50 $\mathrm{mM}$ Tris- $\mathrm{HCl} \mathrm{pH} 7.5,5 \mathrm{mM} \mathrm{KCl}, 5 \mathrm{mM} \mathrm{MgCl}_{2}, 0.5 \mathrm{mM}$ DTT), then resuspended in $20 \mu 1$ of $1 \times$ ubiquitin reaction buffer containing $200 \mathrm{nM}$ UBE1 E1 (R\&D systems, U-110), $1 \mu \mathrm{M}$ UbcH5a E2 (Enzo, BML-UW9050-100), $1 \mu \mathrm{M}$ UbcH5b (Enzo, BML-UW9060-100), 5 mM ATP, $10 \mu \mathrm{M}$ HA-ubiquitin (BostonBiochem, U-110), $10 \mu \mathrm{M}$ MG132, protease inhibitor cocktail, and DMSO or $200 \mu \mathrm{M}$ lenalidomide. Then, in vitro ubiquitination was performed at $30^{\circ} \mathrm{C}$ for $3 \mathrm{~h}$, the proteins were denatured in $2 \% \mathrm{SDS}$ by boiling at $95^{\circ} \mathrm{C}$ for $15 \mathrm{~min}$. The proteins were diluted 20-fold with IP Lysis buffer (Pierce) and immunoprecipitated anti- 
AGIA-conjugated magnetic beads (produced in our laboratory) at $4^{\circ} \mathrm{C}$ for $4 \mathrm{~h}$. The beads were washed four times with $800 \mu 1$ of IP Lysis buffer (Pierce) and the proteins were eluted with $750 \mu 1$ (lane 1-4) or $20 \mu 1$ (lane 5-6) of $1 \times$ sample buffer. After elution, proteins were transferred to another tube, 2-mercaptoethanol (final concentration is 5\%) was added, and were boiled at $98^{\circ} \mathrm{C}$ for $5 \mathrm{~min}$. The proteins were then analysed by immunoblot.

\section{Plzf knockdown experiment in DF-1 cells. To confirm efficiency of shRNA, DF-1 cells} were cultured in a 48-well plate and transfected with $50 \mathrm{ng}$ pcDNA3.1(+)-AGIA-Ggplzf and $400 \mathrm{ng}$ shRNA vector. After $6 \mathrm{~h}$, culture medium was exchanged with new culture medium and the DF-1 cells were harvested after $48 \mathrm{~h}$ of transfection. The lysates were denatured by boiling at $98^{\circ} \mathrm{C}$ for 5 min and analysed by immunoblot.

Animals. Fertilized eggs of white leghorn chicken (Gallus gallus domesticus) were purchased from a domestic poultry farm (Kakeien, Sendai, Japan). Eggs were incubated at $38^{\circ} \mathrm{C}$ until appropriate developmental stage. Embryos were staged according to the criteria made by Hamburger and Hamilton ${ }^{44}$. All animal experiments were properly conducted in accordance with the guidelines of Tohoku University.

Knockdown of Plzf in chicken embryos. $5 \mu \mathrm{g}$ of RCAN(A) retrovirus vector containing chick U6 promoter which expresses shRNA for $g f p$ or $P l z f$ was transfected into M/O chicken strain-derived embryonic fibroblast cells (CEF) cultured in 60-mm dish using Fugene HD transfection regent (Promega). CEF was cultured in DMEM-high glucose containing 10\% FBS, $2 \%$ chicken serum, 1\% penicillin-streptomycin, and spread three times. After ten 10$\mathrm{cm}$ dishes reached to the confluent, medium was changed into DMEM-high glucose containing 2\% FBS and maintained for 24 hours. Then, supernatant was harvested and centrifuged at $100,000 \mathrm{~g}$ for 3 hours at $4^{\circ} \mathrm{C}$ to concentrate retrovirus virion. Isolated virus virion was stored at $-80^{\circ} \mathrm{C}$. To infect retrovirus into the chick embryo, virus virion was sprinkled on the blastderm cells at St. $8 \mathrm{M} / \mathrm{O}$ chick embryo and incubated for 5 days. Fertilized M/O chicken eggs were provided from the National BioResource Project (NBRP) "Chicken/Quail" in Nagoya University.

Thalidomide treatment with chicken embryos. A solid crystal of thalidomide (Tokyo Chemical Industry Co., Ltd) was resolved in 45\% 2-hydroxypropyl-beta-cyclodextrin (HBC, 
760

761

762

763

764

765

766

767

768

769

770

771

772

773

774

775

776

777

778

779

780

781

782

783

784

785

786

787

788

789

790

791

792

FUJIFILM Wako Pure Chemical) for $1-2 \mathrm{~h}$ at $60^{\circ} \mathrm{C}$ to make $2 \mu \mathrm{g} / \mu \mathrm{l}$ thalidomide or 5hydroxythalidomide stock solution. This stock was mixed with same volume of $2 \times$ Hanks buffer as a working solution $(1 \mu \mathrm{g} / \mu \mathrm{l})$. To apply thalidomide to an embryo, a small hole was opened at amnion above a right forelimb bud at Hamburger and Hamilton (HH) st. 18, and working solution was injected in a space between amnion and a right forelimb bud. In the case of samples for in situ hybridization, immunofluorescence, and immunoblot analysis, embryos were treated by $30 \mu \mathrm{l}$ of working solution, and incubated until HH st. 22/23. In the case of samples of skeletal pattern analysis, embryos were treated by $10 \mu$ l of working solution 3 times totally every $12 \mathrm{~h}$ for reducing lethality, and they were incubated until approximately $\mathrm{HH}$ st. 36 (embryonic day 10).

In situ hybridization. Embryos for whole-mount in situ hybridization and fluorescent in situ hybridization in sections (FISH) were fixed by 4\% paraformaldehyde (PFA)/phosphate buffered saline (PBS) at $4^{\circ} \mathrm{C}$ for $12 \mathrm{~h}$. Digoxigenin (DIG)-labeled RNA probes were prepared according to the manufacturer's instructions (Roche). Whole-mount in situ hybridization was performed as previously described ${ }^{45}$. In FISH, $10 \mu \mathrm{m}$ thick-frozen sections were prepared with cryostat (LEICA CM3050S). FISH protocol was described previously ${ }^{46}$, with additional processes in order to amplify fluorescent signal. Additional processes are as follows. After the reaction between DIG-labeled RNA and anti-DIG antibody conjugated horseradish peroxidase (HRP) (Roche), sections were washed 3 times by TNT buffer (100 mM Tris-HCl, $\mathrm{pH} 7.5,150 \mathrm{mM} \mathrm{NaCl}, 0.05 \%$ Tween20), and were treated with DIG amplification working solution in TSA plus DIG Kit (PerkinElmer) for 5 minutes at room temperature (RT) in accordance with manufacturer's instructions (PerkinElmer). After 3 times wash by TNT, samples were treated by anti-DIG antibody conjugated HRP at $4^{\circ} \mathrm{C}$ for $12 \mathrm{~h}$ again.

Immunofluorescence staining. Chicken embryos for Immunofluorescence staining were fixed by $4 \% \mathrm{PFA} / \mathrm{PBS}$ at $4^{\circ} \mathrm{C}$ for $12 \mathrm{~h}$. $10 \mu \mathrm{m}$ thick-frozen sections were prepared with cryostat. PLZF immunofluorescence staining was performed in accordance with Saito et al. ${ }^{47}$, using anti-PLZF rabbit pAb (1:250; GeneTex, GTX111046) as primary antibodies, and antirabbit conjugated HRP (1:500; GE Healthcare) as secondary antibodies. To detect fluorescent signals, we used the TSA Plus Fluorescent System (PerkinElmer) for $5 \mathrm{~min}$ at room temperature. The sections were stained with DAPI (Wako Pure Chemical Corporation) and finally sealed by FluorSave (Calbiochem). 
793 SALL4 was detected as follows. After pre-blocking with blocking solution (1\% blocking 794 reagent in TNT) for $1 \mathrm{~h}$ at RT, the sections were incubated at $4^{\circ} \mathrm{C}$ overnight using an anti795 SALL4 rabbit pAb (1:250; Abcam, ab29112). After three washes in TNT, the specimens 796 were reacted with anti-rabbit IgG-Alexa Fluor 488-conjugated antibody (donkey, Invitrogen) 797 diluted 1:500 with blocking solution for $1 \mathrm{~h}$ at RT, followed by washing three times in TNT. 798 The sections were stained with DAPI and sealed with FluorSave reagent.

799

800 Limb skeletal staining. Embryos for skeletal staining (alcian or Victoria blue staining) were 801 fixed by $10 \%$ formalin/tyrode. Alcian blue staining protocol was described previously ${ }^{48}$. 802 Embryos were harvested in PBS and fixed in 10\% formalin solution overnight. Fixed 803 embryos were washed with $3 \% \mathrm{HCl} / 70 \% \mathrm{EtOH}$ solution for 3 times and stained in $1 \%$ Victoria 804 blue B (Sigma) dissolved in $3 \% \mathrm{HCl} / 70 \% \mathrm{EtOH}$ overnight. Embryos were washed with $8053 \% \mathrm{HCl} / 70 \% \mathrm{EtOH}$ solution overnight, then they were treated in methylsalicylate for 806 transparent process.

807

808 Imaging. Images of FISH and immunofluorescence staining on sections were obtained using 809 a TCS SP5 confocal microscope (LEICA). Images of whole-mount in situ hybridization were 810 obtained using a fluorescent stereo microscope (LEICA M165FC with Olympus DP74 811 camera). Images of skeletal staining were obtained using a stereo microscope (Olympus 812 SZX16 with Olympus DP21 camera).

814 Statistical analysis. Significant changes were analysed by a one-way or two-way ANOVA 815 followed Tukey's post-hoc test using Graph Pad Prism 8 software (GraphPad, Inc.). For all 816 tests, a $P$ value of less than 0.05 was considered statistically significant.

818 Data availability. The authors declare that all data supporting the findings of this study are 819 available in the manuscript and its supplementary files or are available from the 820 corresponding author upon reasonable request. 


\section{References}

823 1. Miller, M. T. \& Strömland, K. Teratogen update: thalidomide: a review, with a focus on 824 ocular findings and new potential uses. Teratology 60, 306-321 (1999).

825

826

827

828

829

830

831

832

833

834

835

836

837

838

839

840

841

842

843

844

845

846

847

848

849

850

851

852

853

854

2. Smithells, R. W. \& Newman, C. G. Recognition of thalidomide defects. J. Med. Genet. 29, 716-723 (1992).

3. Vargesson, N. Thalidomide embryopathy: an enigmatic challenge. ISRN Dev Biol. 2013, 1-18 (2013).

4. Ito, T. et al. Identification of a primary target of thalidomide teratogenicity. Science $\mathbf{3 2 7}$, 1345-1350 (2010).

5. Krönke, J. et al. Lenalidomide causes selective degradation of IKZF1 and IKZF3 in multiple myeloma cells. Science 343, 301-305 (2014).

6. Lu, G. et al. The myeloma drug lenalidomide promotes the cereblon-dependent destruction of Ikaros proteins. Science 343, 305-309 (2014).

7. Krönke, J. et al. Lenalidomide induces ubiquitination and degradation of CK1 $\alpha$ in $\operatorname{del}(5 q)$ MDS. Nature 523, 183-188 (2015).

8. Matyskiela, M. E. et al. A novel cereblon modulator recruits GSPT1 to the CRL4(CRBN) ubiquitin ligase. Nature 535, 252-257 (2016).

9. Donovan, K. A. et al. Thalidomide promotes degradation of SALL4, a transcription factor implicated in Duane Radial syndrome. eLife 7, e38430 (2018).

10. Matyskiela, M. E. et al. SALL4 mediates teratogenicity as a thalidomide-dependent cereblon substrate. Nat. Chem. Biol. 14, 981-987 (2018).

11. Kohlhase, J. et al. SALL4 mutations in Okihiro syndrome (Duane-radial ray syndrome), acro-renal-ocular syndrome, and related disorders. Hum. Mutat. 26, 176-183 (2005).

12. Akiyama, R. et al. Sall4-Gli3 system in early limb progenitors is essential for the development of limb skeletal elements. Proc. Natl. Acad. Sci. U.S.A. 112, 5075-5080 (2015).

13. Suliman, B. A., Xu, D. \& Williams, B. R. G. The promyelocytic leukemia zinc finger protein: two decades of molecular oncology. Front Oncol 2, 74 (2012).

14. Barna, M., Hawe, N., Niswander, L. \& Pandolfi, P. P. Plzf regulates limb and axial skeletal patterning. Nat. Genet. 25, 166-172 (2000).

15. Barna, M., Pandolfi, P. P. \& Niswander, L. Gli3 and Plzf cooperate in proximal limb patterning at early stages of limb development. Nature 436, 277-281 (2005).

16. Fischer, S. et al. Biallelic loss of function of the promyelocytic leukaemia zinc finger 
(PLZF) gene causes severe skeletal defects and genital hypoplasia. J. Med. Genet. 45, 731-737 (2008).

17. Sievers, Q. L. et al. Defining the human $\mathrm{C} 2 \mathrm{H} 2$ zinc finger degrome targeted by thalidomide analogs through CRBN. Science 362, (2018).

18. Ando, Y., Fuse, E. \& Figg, W. D. Thalidomide metabolism by the CYP2C subfamily. Clinical Cancer Research 8, 1964-1973 (2002).

19. Chowdhury, G. et al. Human liver microsomal cytochrome P450 3A enzymes involved in thalidomide 5-hydroxylation and formation of a glutathione conjugate. Chem. Res. Toxicol. 23, 1018-1024 (2010).

20. Yamamoto, T. et al. Synthesis, configurational stability and stereochemical biological evaluations of (S)- and (R)-5-hydroxythalidomides. Bioorg. Med. Chem. Lett. 19, 39733976 (2009).

21. Fischer, E. S. et al. Structure of the DDB1-CRBN E3 ubiquitin ligase in complex with thalidomide. Nature 512, 49-53 (2014).

22. Mori, T. et al. Structural basis of thalidomide enantiomer binding to cereblon. Sci Rep $\mathbf{8}$, 1294 (2018).

23. Therapontos, C., Erskine, L., Gardner, E. R., Figg, W. D. \& Vargesson, N. Thalidomide induces limb defects by preventing angiogenic outgrowth during early limb formation. Proc. Natl. Acad. Sci. U.S.A. 106, 8573-8578 (2009).

24. Lepper, E. R., Smith, N. F., Cox, M. C., Scripture, C. D. \& Figg, W. D. Thalidomide metabolism and hydrolysis: mechanisms and implications. Curr. Drug Metab. 7, 677685 (2006).

25. Kazuki, Y. et al. Thalidomide-induced limb abnormalities in a humanized CYP3A mouse model. Sci Rep 6, 21419 (2016).

26. Sawasaki, T., Ogasawara, T., Morishita, R. \& Endo, Y. A cell-free protein synthesis system for high-throughput proteomics. Proc. Natl. Acad. Sci. U.S.A. 99, 14652-14657 (2002).

27. Tadokoro, D. et al. Characterization of a caspase-3-substrate kinome using an N- and Cterminally tagged protein kinase library produced by a cell-free system. Cell Death Dis 1, e89-e89 (2010).

28. Takahashi, H. et al. Establishment of a Wheat Cell-Free Synthesized Protein Array Containing 250 Human and Mouse E3 Ubiquitin Ligases to Identify Novel Interaction between E3 Ligases and Substrate Proteins. PLoS ONE 11, e0156718 (2016). 
888

889

890

891

892

893

894

895

896

897

898

899

900

901

902

903

904

905

906

907

908

909

910

911

912

913

914

915

916

917

918

919

920

29. Nemoto, K. et al. Tyrosine phosphorylation of the GARU E3 ubiquitin ligase promotes gibberellin signalling by preventing GID1 degradation. Nature Communications 8, 1004 (2017).

30. Hideshima, T. et al. Thalidomide and its analogs overcome drug resistance of human multiple myeloma cells to conventional therapy. Blood 96, 2943-2950 (2000).

31. Yano, T. et al. AGIA Tag System Based on a High Affinity Rabbit Monoclonal Antibody against Human Dopamine Receptor D1 for Protein Analysis. PLoS ONE 11, e0156716 (2016).

32. An, J. et al. pSILAC mass spectrometry reveals ZFP91 as IMiD-dependent substrate of the CRL4CRBN ubiquitin ligase. Nature Communications 8, 15398 (2017).

33. Liška, F. et al. Targeting of the Plzf Gene in the Rat by Transcription Activator-Like Effector Nuclease Results in Caudal Regression Syndrome in Spontaneously Hypertensive Rats. PLoS ONE 11, e0164206 (2016).

34. Uhlén, M. et al. A human protein atlas for normal and cancer tissues based on antibody proteomics. Mol. Cell Proteomics 4, 1920-1932 (2005).

35. Xu, D. et al. Promyelocytic leukemia zinc finger protein regulates interferon-mediated innate immunity. Immunity 30, 802-816 (2009).

36. Savage, A. K. et al. The transcription factor PLZF directs the effector program of the NKT cell lineage. Immunity 29, 391-403 (2008).

37. Kovalovsky, D. et al. The BTB-zinc finger transcriptional regulator PLZF controls the development of invariant natural killer T cell effector functions. Nat. Immunol. 9, 10551064 (2008).

38. Serafini, N., Vosshenrich, C. A. J. \& Di Santo, J. P. Transcriptional regulation of innate lymphoid cell fate. Nat. Rev. Immunol. 15, 415-428 (2015).

39. Fink, E. C. et al. CrbnI391V is sufficient to confer in vivo sensitivity to thalidomide and its derivatives in mice. Blood 132, 1535-1544 (2018).

40. Gemechu, Y. et al. Humanized cereblon mice revealed two distinct therapeutic pathways of immunomodulatory drugs. Proc. Natl. Acad. Sci. U.S.A. 115, 11802-11807 (2018).

41. Beckman, R. \& Kampf, H. H. Zur quantitativen Bestimmung und zum qualitativen Nachweis von N-phthalyl-glutaminsauremid (thalidomide). Arzneimittelforschung 11, 45-47 (1961).

42. Sawasaki, T. et al. Arabidopsis HY5 protein functions as a DNA-binding tag for 
purification and functional immobilization of proteins on agarose/DNA microplate. FEBS Lett 582, 221-228 (2008).

43. Nagase, T. et al. Exploration of Human ORFeome- High-Throughput Preparation of ORF Clones and Efficient Characterization of Their Protein Products. DNA Res. 15, 137149 (2008).

44. Hamburger, V. \& Hamilton, H. L. A series of normal stages in the development of the chick embryo. J. Morphol. 88, 49-92 (1951).

45. Tonegawa, A., Funayama, N, Ueno, N., \& Takahashi, Y. Mesodermal subdivision along the mediolateral axis in chicken controlled by different concentrations of BMP-4. Development 124, 1975-84 (1997).

46. Sato, Y. et al. Notch signaling mediates the segmental specification of angioblasts in somites and their directed migration toward the dorsal aorta in avian embryos. Dev. Cell 14, 890-901 (2008).

47. Saito, D., Takase, Y., Murai, H. \& Takahashi, Y. The dorsal aorta initiates a molecular cascade that instructs sympatho-adrenal specification. Science 336, 1578-1581 (2012).

48. Seki, R. et al. AP-2 $\beta$ is a transcriptional regulator for determination of digit length in tetrapods. Dev. Biol. 407, 75-89 (2015).

\section{Acknowledgements}

We thank H. Yamakawa (Kazusa DNA Research Institute) for the vector construction for the human protein array, C. Takahashi and C. Furukawa for technical assistance, and the Applied Protein Research Laboratory of Ehime University. We also thank Prof. F. Tokunaga and Dr. D. Oikawa (Osaka City University) for providing the DLBCL cell lines. This work was mainly supported by the Platform Project for Supporting Drug Discovery and Life Science Research (Basis for Supporting Innovative Drug Discovery and Life Science Research (BINDS)) from AMED under Grant Number JP19am0101077 (H. Takeda, T.S.), a Grant-inAid for Scientific Research on Innovative Areas (JP16H06579 for T.S.) from the Japan Society for the Promotion of Science (JSPS). This work was also partially supported by JSPS KAKENHI (JP17J08477 for S.Y., JP16H04729, JP19H03218 for T.S., JP17H06112 for N.S., and JP18H02446, JP18H04811, JP18H04756 for K.T), a Grant-in-Aid for JSPS Research Fellow (JP17J08477 for S.Y) from JSPS., and Takeda Science Foundation. Establishment 
952 and screening of the human protein array used in this study was conducted in Proteo-Science

953 Center at Ehime University with the support of MEXT.

954

955 Author Contributions

956 S.Y. performed the biochemical, molecular, and cellular biology experiments. T.I. and

957 H.Takeda constructed the human protein array. H.Takahashi supported the screening. E.T.

958 and N.S. synthesized and analysed the 5-hydroxythalidomide. H.M., D.S., G.A., T.Suzuki,

959 and K.T. performed the chicken teratogenesis studies. S.Y. and T.Sawasaki analysed the data,

960 designed the study, wrote the paper, and all authors contributed to the manuscript.

961

962 Additional information

963 Supplementary Information accompanies this paper at XXXXXX.

964

965 Competing interests: The authors declare no competing financial interests.

966

967 Reprints and permission information is available online at XXXXXXX.

968 
a

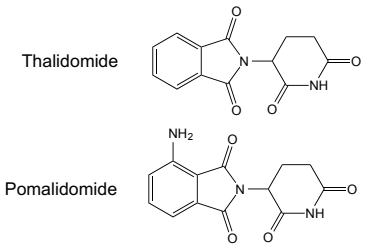

Lenalidomide

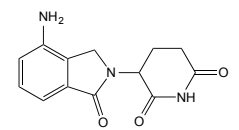

b

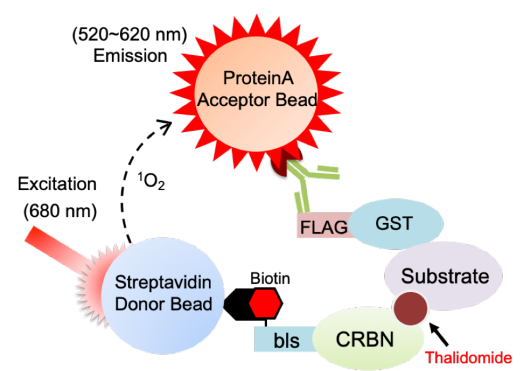

e

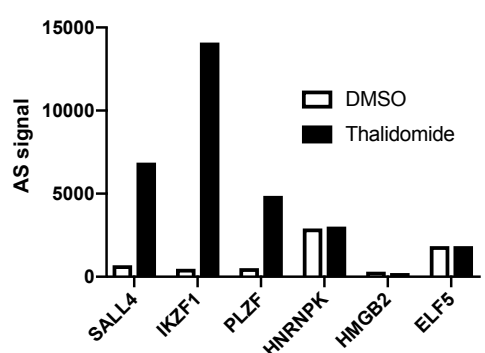

c

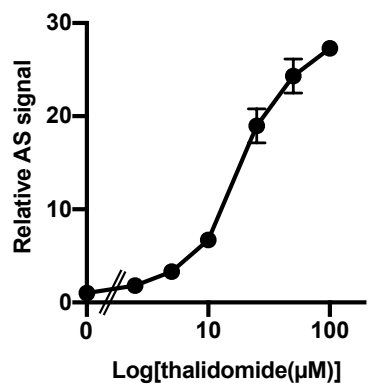

f

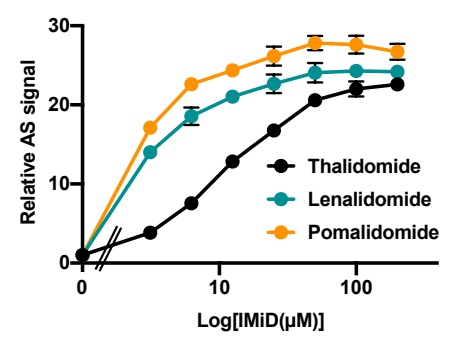

969

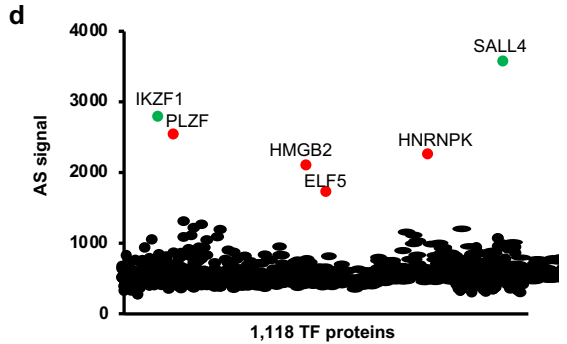

970

971

972

973

974

975

976

977

978

979

980

981

982

983

984

985

986

987

Figure 1. Identification of thalidomide-dependent interactors of CRBN using a cell-free based human TF protein array

a, Chemical structures of thalidomide, pomalidomide and lenalidomide. b, Schematic diagram of the thalidomide-dependent in vitro binding assay between CRBN and substrates using AlphaScreen technology. $\mathbf{c}$, Detection of luminescent signals of thalidomide-dependent interactions between bls-CRBN and FLAG-GST-IKZF1. Dose-dependent signals (DMSO, $2.5,5,10,25,50$, or $100 \mu \mathrm{M}$ thalidomide) was analysed with an in vitro binding assay using AlphaScreen technology. d, Results of in vitro high-throughput screening, targeting 1,118 human transcription factors. Green and red spots denote known neosubstrates and candidate clones, respectively. e, Confirmation of thalidomide-dependency on six hit proteins using an in vitro binding assay. Interaction between bls-CRBN and FLAG-GST-protein in the presence of DMSO or $50 \mu \mathrm{M}$ thalidomide was detected using AlphaScreen technology. f, In vitro binding assay for thalidomide, pomalidomide, and lenalidomide. Interaction between bls-CRBN and FLAG-GST-PLZF in the presence of DMSO, $(3.125,6.25,12.5,25,50,100$, or $200 \mu \mathrm{M}$ ) thalidomide, pomalidomide or lenalidomide was analysed using AlphaScreen technology. All relative AS (AlphaScreen) signals were expressed as relative luminescent signal with luminescent signal of DMSO as one, and error bars mean \pm standard deviation $(n=3)$. 

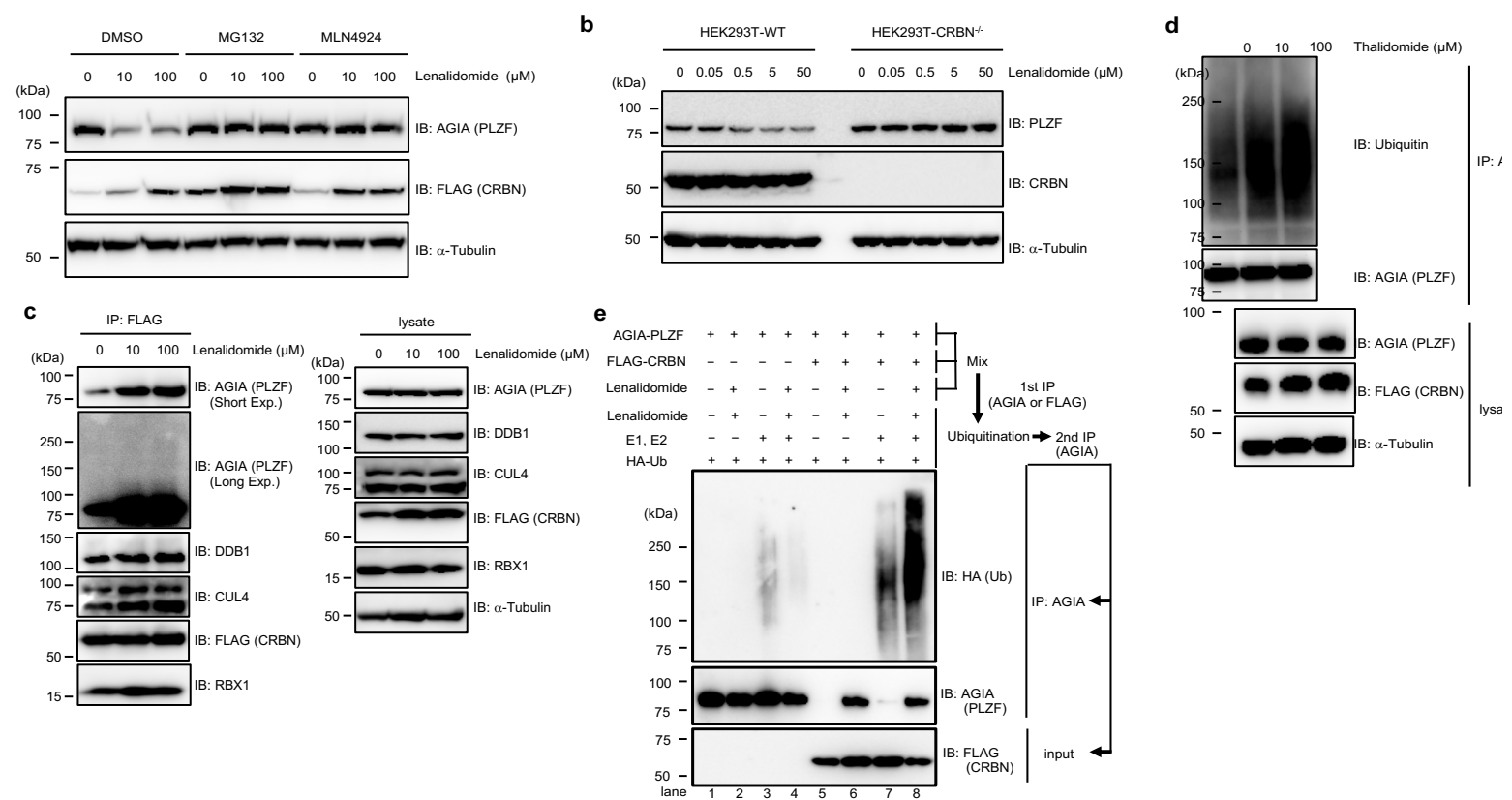

Figure 2. PLZF is a substrate of CRL4 ${ }^{\mathrm{CRBN}}$ with thalidomide and lenalidomide for E3

\section{ubiquitin ligase}

a, Immunoblot analysis of AGIA-PLZF protein levels in AGIA-PLZF and FLAG-CRBN expressing HEK293T cells treated with DMSO or lenalidomide in the presence of DMSO, MG132, or MLN4924 for 9 h. b, Immunoblot analysis of endogenous PLZF protein levels in HEK293T cells or CRBN ${ }^{-/-}$HEK293T cells treated with DMSO or lenalidomide for $24 \mathrm{~h}$. c, Immunoprecipitation of FLAG-CRBN in FLAG-CRBN and AGIA-PLZF expressing HEK293T cells treated with DMSO or lenalidomide in the presence of DMSO or MG132 for $8 \mathrm{~h}$. Components of CRL ${ }^{\text {FLAG-CRBN }}$ and AGIA-PLZF were detected using each specific antibody, as indicated. d, Ubiquitination of AGIA-PLZF in AGIA-PLZF and FLAG-CRBN expressing $\mathrm{CRBN}^{-/} \mathrm{HEK} 293 \mathrm{~T}$ cells treated with DMSO or thalidomide in the presence of DMSO or MG132 for $10 \mathrm{~h}$. AGIA-PLZF was immunoprecipitated using anti-AGIA antibody and the polyubiquitin chain on AGIA-PLZF was analysed by immunoblot. e, In vitro binding and ubiquitination assay of AGIA-PLZF. Empty vector, AGIA-PLZF, or FLAG-CRBN expressing HEK293T cells were lysed and the lysates were mixed. The first immunoprecipitation with anti-AGIA or anti-FLAG antibodies was performed in the presence of DMSO or $200 \mu \mathrm{M}$ lenalidomide. The purified AGIA-PLZF or CRL4 ${ }^{\text {FLAG-CRBN }}$ complex, including AGIA-PLZF and FLAG-CRBN, was incubated with recombinant E1, E2, and HA-ubiquitin in the presence of DMSO or $200 \mu \mathrm{M}$ lenalidomide, and the second immunoprecipitation was performed using anti-AGIA antibody. Ubiquitination of PLZF was analysed by immunoblot. 
a

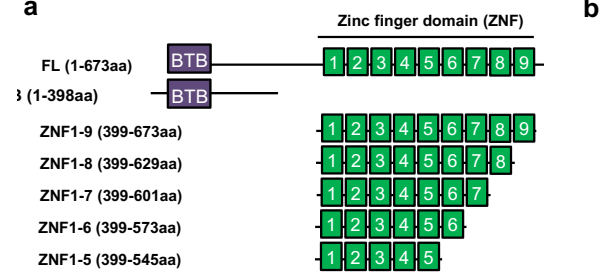

ZNF1-5 (399-545aa)

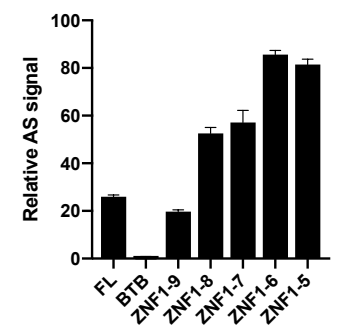

c

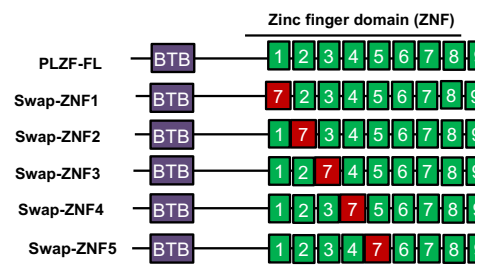

d

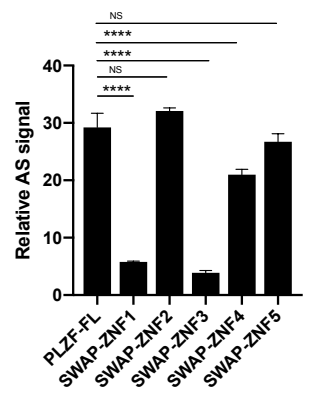

1010 Figure 3. Interaction regions in PLZF for binding to CRBN with thalidomide

1011 a, Schematic diagram of PLZF and truncated PLZFs. b, In vitro binding assay using truncated 1012 PLZF. Thalidomide-dependent interaction between bls-CRBN and FLAG-GST-PLZF-full 1013 length (FL) or truncated FLAG-GST-PLZF was analysed in the presence of DMSO or 50 $1014 \mu \mathrm{M}$ thalidomide using AlphaScreen technology. c, Schematic diagram of swapped PLZF

1015 mutants. d, In vitro binding assay using swapped PLZF mutants was performed using the 1016 same procedure as in Figure 3b. e, Amino acid sequences of ZNF1 and ZNF3 in PLZF. f, In vitro binding assay using point mutants of PLZF was performed using the same procedure as in Figure 3b. g, Immunoblot analysis of AGIA-PLZF protein levels in FLAG-CRBN and 1019 PLZF-WT, PLZF-ZNF1-GA, PLZF-ZNF3-GA, or PLZF-ZNF1,3-GA expressing CRBN-HEK293T cells treated with DMSO or thalidomide for $16 \mathrm{~h}$. All relative AS (AlphaScreen) signals were expressed as relative luminescent signal with luminescent signal of DMSO as one. Error bars mean \pm standard deviation $(\mathrm{n}=3)$ and $P$ values were calculated by one-way ANOVA with Tukey's post-hoc test (NS $=$ Not Significant, and $\left.{ }^{* * * *} P<0.0001\right)$. 
a

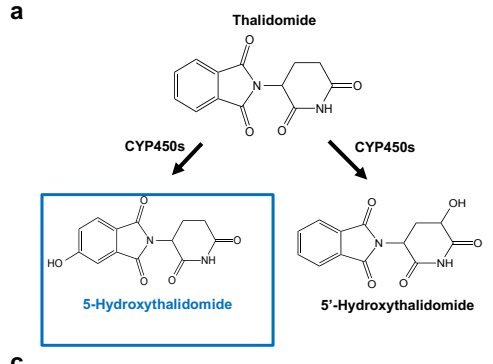

c

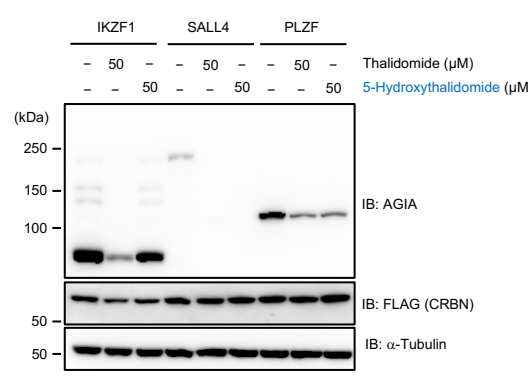

b

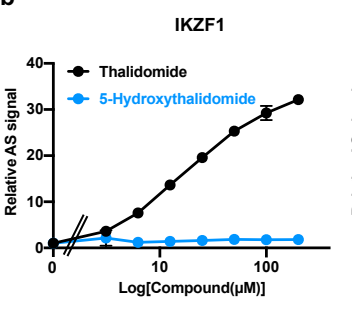

d
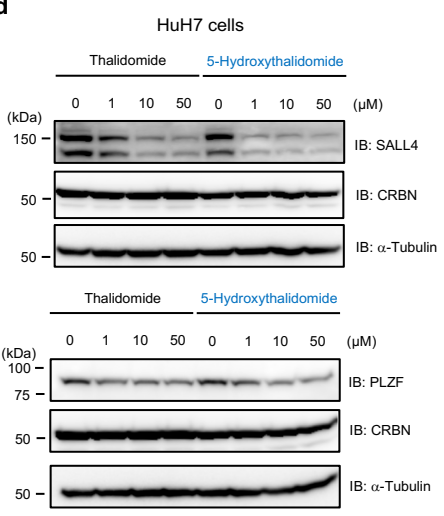

SALL4

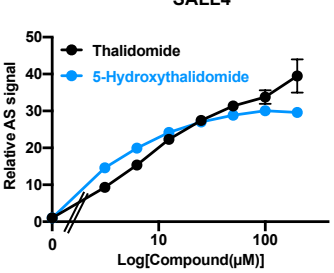

e

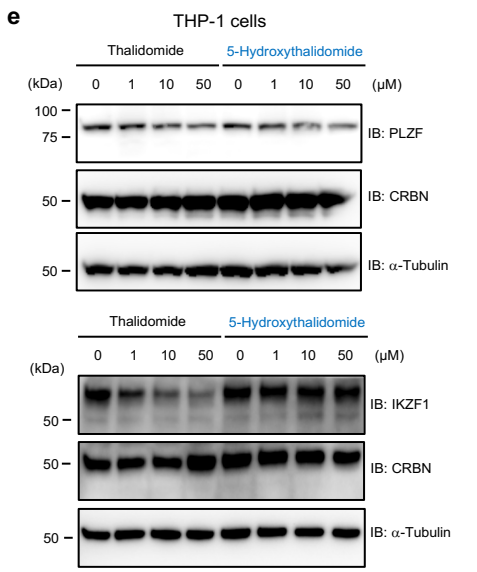

1025

1026

1027

1028

1029

1030

1031

1032

1033

1034

1035

1036

Figure 4. 5-Hydroxythalidomide induces degradation of PLZF and SALL4 by CRBN

a, Schematic diagram of thalidomide metabolites by CYPs. b, In vitro binding assay for thalidomide and 5-hydroxythalidomide. Interaction between bls-CRBN and FLAG-GSTIKZF1, -SALL4, -PLZF in the presence of DMSO, thalidomide or 5-hydroxythalidomide $(3.125,6.25,12.5,25,50,100$, or $200 \mu \mathrm{M})$ was analysed using AlphaScreen technology. c, Immunoblot analysis of AGIA-PLZF, AGIA-SALL4, or AGIA-PLZF in FLAG-CRBN expressing $\mathrm{CRBN}^{-/}$HEK293T cells treated with DMSO, thalidomide, or 5hydroxythalidomide for $16 \mathrm{~h}$. d, Immunoblot analysis of endogenous SALL4 or PLZF protein levels in $\mathrm{HuH} 7$ cells treated with DMSO, thalidomide, or 5-hydroxytahalidomide for $24 \mathrm{~h}$. e, Immunoblot analysis of endogenous PLZF or IKZF1 protein levels in THP-1 cells treated with DMSO, thalidomide, or 5-hydroxytahalidomide for $24 \mathrm{~h}$. 
a

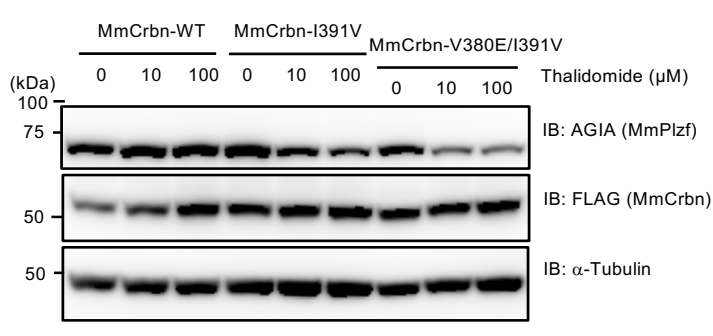

C

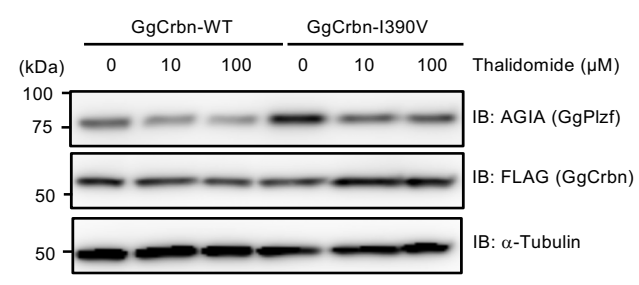

e

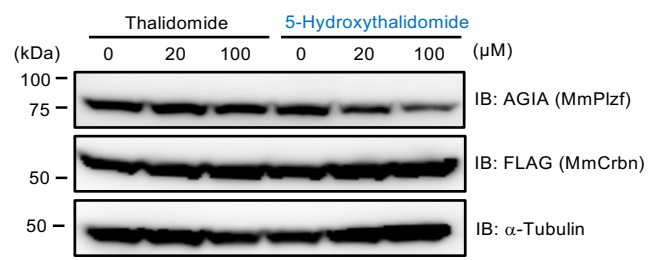

g

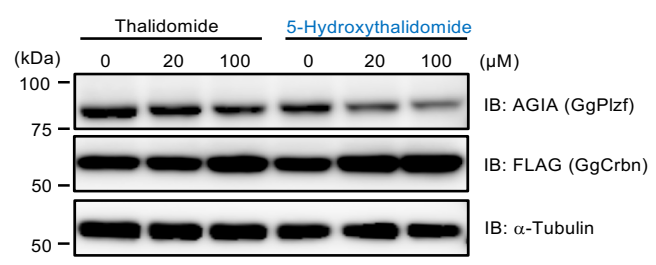

b

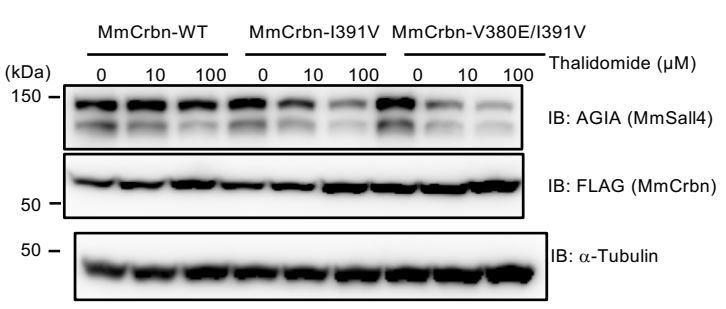

d

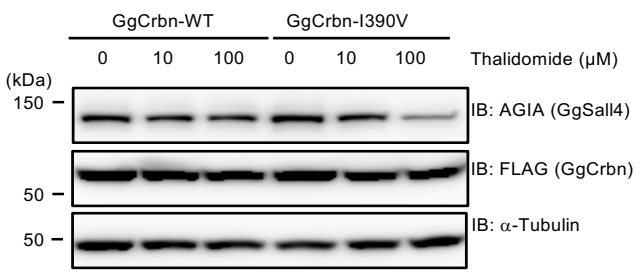

f

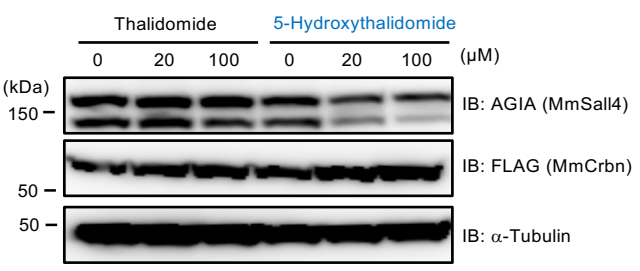

h

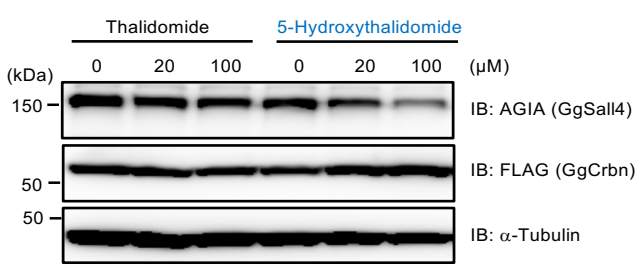

Figure 5. Crbn-dependent degradation of Plzf and Sall4 from mouse and chicken by treatment with thalidomide and 5-hydroxythalidomide MmCrbn-I391V or -MmCrbn-V380E/I391V expressing $\mathrm{CRBN}^{-/-} \mathrm{HEK}^{293 \mathrm{~T}}$ cells treated

1043 with DMSO or thalidomide for 16 h. c, d, Immunoblot analysis of AGIA-GgPlzf (c) or -

1044 GgSall4 (d) in FLAG-GgCrbn-WT or -GgCrb-I390V expressing CRBN ${ }^{-/}$HEK293T cells treated with DMSO or thalidomide for $16 \mathrm{~h}$. e, f, Immunoblot analysis of AGIA-MmPlzf (e) or -MmSall4 (f) in FLAG-MmCrbn-WT expressing $\mathrm{CRBN}^{-/-}$HEK293T cells treated with

1047 indicated concentration of DMSO, thalidomide or 5-hydroxythalidomide for $16 \mathrm{~h}$. $\mathbf{g}, \mathbf{h}$, 1048 Immunoblot analysis of AGIA-GgPlzf (g) or -GgSall4 (h) in FLAG-GgCrbn-WT expressing 
$1049 \mathrm{CRBN}^{-/-} \mathrm{HEK} 293 \mathrm{~T}$ cells treated with indicated concentration of DMSO, thalidomide or 51050 hydroxythalidomide for $16 \mathrm{~h}$. 
a

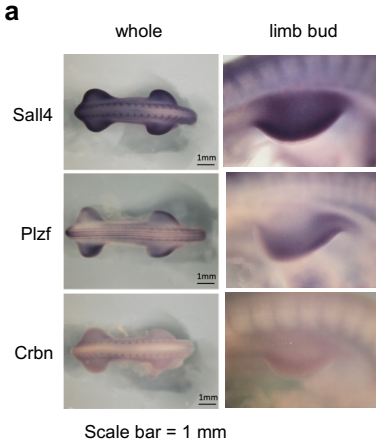

d

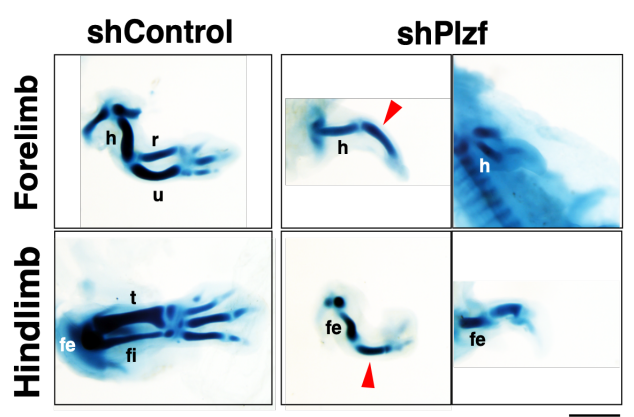

b

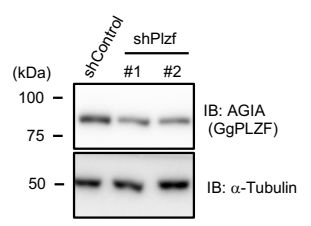

1052

1053

1054

1055

1056

1057

1058

1059

1060

1061

1062

1063

1064

1065 embryos in Fig. 6d. c
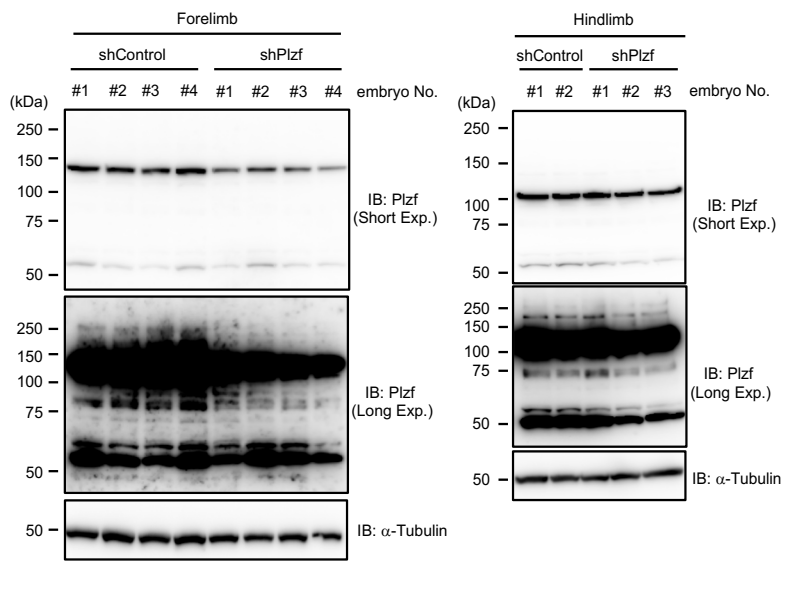

\begin{tabular}{lcc} 
& shControl $(n=10)$ & shPIzf $(n=9)$ \\
\hline Shortened forelimb & 0 & 7 \\
Shortened hindlimb & 0 & 8 \\
Reduction of digit number & 0 & 3 \\
Reduction of zeugopod bone & 0 & 3
\end{tabular}

Figure 6. Downregulation of Plzf causes abnormal limb development in chicken embryo a, Sall4, Plzf or Crbn mRNA expression in E4 chicken embryos was analysed by wholemount in situ hybridization. Left panel shows whole chicken embryo and right panel shows right forelimb bud. b, Immunoblot analysis of AGIA-GgPlzf in AGIA-GgPlzf expressing DF-1 cells transfected with shContol (shGFP) or shPlzf expression vector. c, Immunoblot analysis of Plzf from tissue of chicken forelimb or hindlimb bud. Endogenous Plzf protein expression was detected by immunoblot using chicken embryos infected with RCAN virus packaging shControl or shPlzf (forelimb shControl $(n=4)$, forelimb shPlzf $(n=4)$, hindlimb shControl $(n=2)$ or hindlimb shPlzf $(n=4))$. d, Limb skeletal stained with Victoria blue. Skeletal patterning of forelimb and hindlimb in E6 chicken embryos infected RCAN virus packaging shControl $(n=10)$ or shPlzf $(n=9)$ were analysed by Victoria blue staining. $h$; humerus, r; radius, u; ulna, fe; femur, fi; fibula, t; tibia. e, Teratogenic phenotypes of chicken 


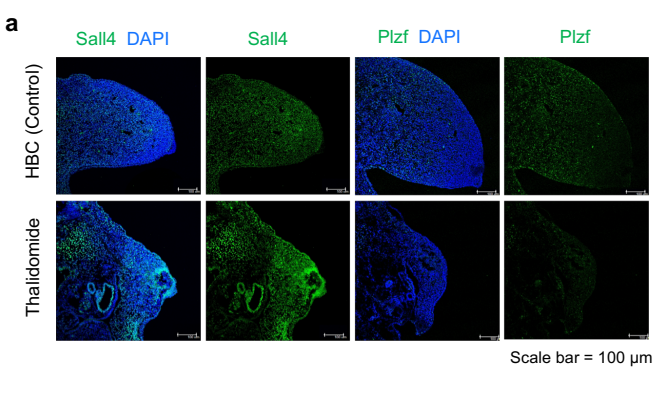

d
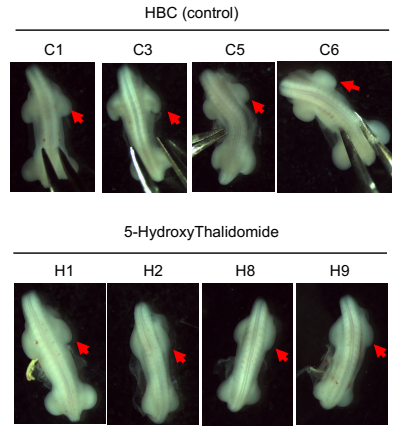

D
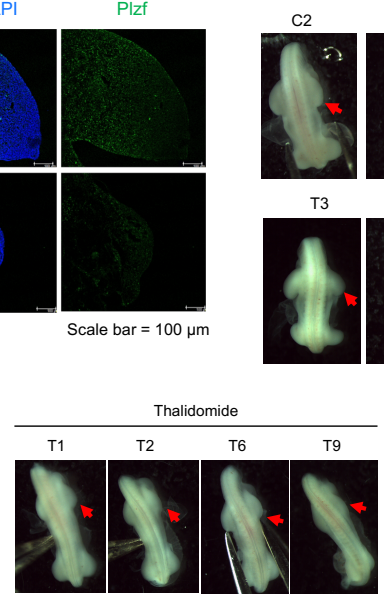

1066

1067

1068

1069

1070

1071

1072

1073

1074

1075

1076

1077

1078

\section{Figure 7. Thalidomide induces degradation of PLZF in abnormal chick limb buds}

a, Immunohistochemical staining of Sall4 or Plzf in chicken forelimb bud. Endogenous Sall4 or Plzf protein expression was detected using forelimb bud section in chicken embryos treated with $\mathrm{HBC}(\mathrm{n}=4)$ or $1 \mu \mathrm{g} / \mu \mathrm{l}$ thalidomide $(\mathrm{n}=4)$. b. Photographs show chicken embryos treated with HBC (control, $\mathrm{n}=6, \mathrm{C} 2, \mathrm{C} 3$, and C5) or strong phenotype (1 $\mu \mathrm{g} / \mu 1$ thalidomide, $\mathrm{n}=18, \mathrm{~T} 3, \mathrm{~T} 8, \mathrm{~T} 18)$ corresponding to immunoblot analysis in Figure 7c. Red arrows show treated regions. c, Endogenous Plzf or Sall4 protein expression in chicken embryos in Fig. $7 \mathrm{~b}$ was detected by immunoblot. d, Photographs show chicken embryos treated with $\mathrm{HBC}$ (control, $\mathrm{n}=10, \mathrm{C} 1, \mathrm{C} 3, \mathrm{C} 5$ and C6), $1 \mu \mathrm{g} / \mu 1$ thalidomide ( $\mathrm{n}=11, \mathrm{~T} 1, \mathrm{~T} 2$, T6, T9) or $1 \mu \mathrm{g} / \mu 1$ 5-hydroxythalidomide ( $\mathrm{n}=10, \mathrm{H} 1, \mathrm{H} 2, \mathrm{H} 8, \mathrm{H} 9)$ corresponding to immunoblot analysis in Figure 6d. Red arrows show treated regions. e, Endogenous Plzf or Sall4 protein expression in chicken embryos in Fig. $7 \mathrm{~d}$ was detected by immunoblot. 
a

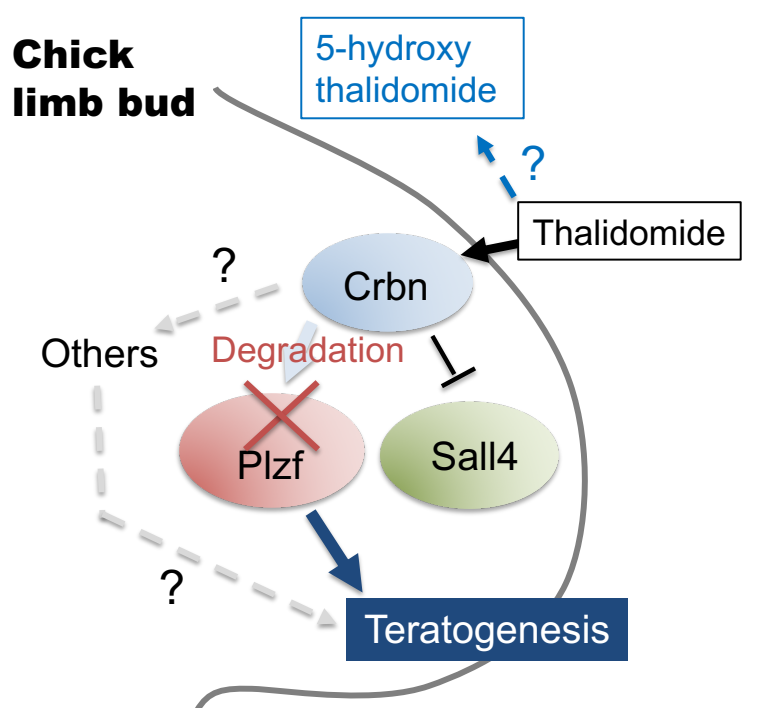

b

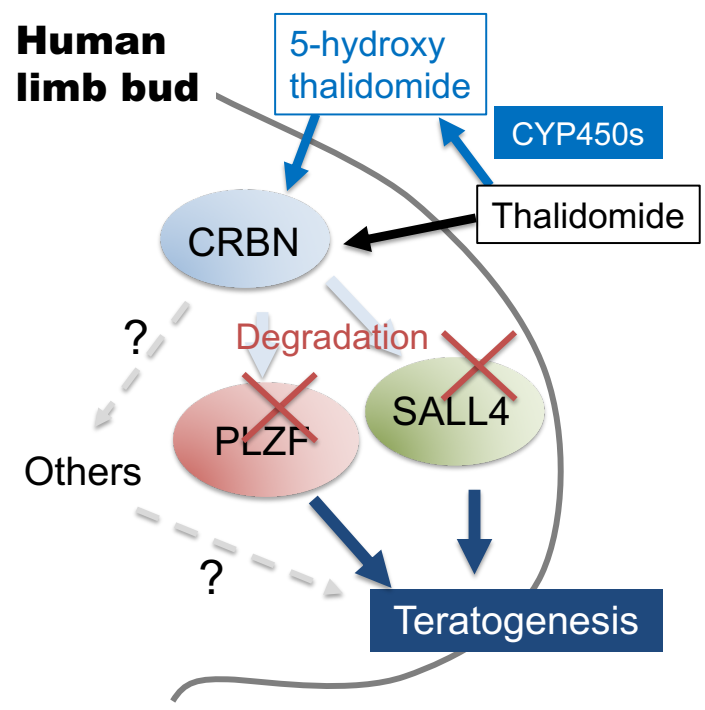

1079 1080 1081 1082 1083

Figure 8. Model cartoon of thalidomide-induced teratogenicity in chicken or human limb bud

a, Model of thalidomide teratogenesis in chicken limb bud. b, Model of thalidomide teratogenesis in human limb bud. 